Illinois State University

ISU ReD: Research and eData

Theses and Dissertations

4-16-2021

\title{
Exploring African American Vernacular English and Disproportionality in Special Education
}

Camille O'Quin

Illinois State University, camille.oquin@gmail.com

Follow this and additional works at: https://ir.library.illinoisstate.edu/etd

\section{Recommended Citation}

O'Quin, Camille, "Exploring African American Vernacular English and Disproportionality in Special Education" (2021). Theses and Dissertations. 1459.

https://ir.library.illinoisstate.edu/etd/1459

This Dissertation is brought to you for free and open access by ISU ReD: Research and eData. It has been accepted for inclusion in Theses and Dissertations by an authorized administrator of ISU ReD: Research and eData. For more information, please contact ISUReD@ilstu.edu. 


\section{EXPLORING AFRICAN AMERICAN VERNACULAR ENGLISH AND \\ DISPROPORTIONALITY IN SPECIAL EDUCATION}

\section{CAMILLE BYRD O’QUIN}

\section{Pages}

More than $80 \%$ of African Americans in the United States of America speak the dialect African American Vernacular English (AAVE) (Green, 2002); however, misperceptions of AAVE may have a direct impact on African American students' equitable access to education in United States public schools (Beneke et al., 2015). African Americans are disproportionality over-represented in special education in disability categories that require subjective clinical judgements, including specific learning disabilities. Numerous factors may contribute to this phenomenon, including the subjective process for special education referrals (Herzik, 2015) and teachers' biased perceptions of cultural differences, including students' use of AAVE (Gupta, 2010). The need for educators to build cultural competence and gain knowledge about cultural communication patterns like AAVE is discussed. The purpose of this research study was to investigate the special education referral process amongst teachers, administrators, and support personnel for third graders who communicate using AAVE in writing. A mixed method concurrent design was used for this study (Creswell et al., 2017). Results suggest that vignettes written in AAVE were referred for evaluation 6.5 times more frequently than vignettes written in MAE. Referrals were only made by general education classroom teachers and speech-language pathologists. Educators listed a variety of reasons for making decisions regarding referral to special education, including diversity, dialect, and AAVE. Implications include the need to 
develop a more complete understanding of choices made for a special education referral that may impact AAVE speaking students and disproportionality in special education.

KEYWORDS: African American Vernacular English, bias, bidialectialism, codeswitching, perception, culturally responsive pedagogy, disproportionality, special education, referral 


\section{EXPLORING AFRICAN AMERICAN VERNACULAR ENGLISH AND DISPROPORTIONALITY IN SPECIAL EDUCATION}

CAMILLE BYRD O'QUIN

A Dissertation Submitted in Partial

Fulfillment of the Requirements for the Degree of

DOCTOR OF EDUCATION

Department of Special Education

ILLINOIS STATE UNIVERSITY 
(C) 2021 Camille Byrd O'Quin 


\section{EXPLORING AFRICAN AMERICAN VERNACULAR ENGLISH AND DISPROPORTIONALITY IN SPECIAL EDUCATION}

CAMILLE BYRD O'QUIN

COMMITTEE MEMBERS:

Mark Zablocki, Chair

Debbie Shelden

Carrie Anna Courtad

Lydia Kyei-Blankson 


\section{ACKNOWLEDGMENTS}

I must start by acknowledging my faithful, loving family: my husband, Troy, and my twin daughters, Abigail and Sophia. You have supported me for years and years throughout this process, patiently waiting for my undivided attention to return to you. I love you all so very much and I am looking forward to spending more time with you again. Also, I could not have completed this process without the constant guidance of my committee. Thank you, Mark, Lydia, Carrie Anna, and Debbie. Thank you to Sarah Jozwik and April Mustian, too, for your friendship, support, and encouragement; you have truly been a lifeline throughout this process. Lastly, thank you to my parents for giving me an office space where I could write during the COVID-19 Pandemic. I dare say I would not have finished writing this document without it.

C.B.O. 


\section{CONTENTS}

Page

ACKNOWLEDGMENTS

CONTENTS

TABLES Di

CHAPTER I: INTRODUCTION 1

Misperception of Cultural Linguistic Difference as a Disorder 3

Misperception of AAVE as a Disorder 4

Description of AAVE Instruction Parallel to United States Public School Norms 5

Cultural Linguistic Mismatch $\quad 5$

$\begin{array}{ll}\text { Solutions to Cultural Linguistic Mismatch } & 6\end{array}$

$\begin{array}{ll}\text { Linguistic Properties of AAVE } & 7\end{array}$

$\begin{array}{ll}\text { The Role of Codeswitching } & 8\end{array}$

AAVE, Codeswitching, and Education $\quad 9$

$\begin{array}{ll}\text { Remedies to School Norms Disruption } & 10\end{array}$

Statement of the Problem 13

$\begin{array}{ll}\text { Disproportionality in Special Education } & 13\end{array}$

$\begin{array}{ll}\text { Policy Related to Disproportionality } & 14\end{array}$

$\begin{array}{ll}\text { Purpose of the Study } & 15\end{array}$

$\begin{array}{ll}\text { Significance of the Study } & 16\end{array}$

$\begin{array}{ll}\text { Positionality } & 16\end{array}$

$\begin{array}{ll}\text { Summary } & 18\end{array}$

CHAPTER II: LITERATURE REVIEW 20 
$\begin{array}{ll}\text { A Review of the Literature } & 20\end{array}$

$\begin{array}{ll}\text { Perception of AAVE } & 23\end{array}$

Over-referral of African American Students to Special Education 29

$\begin{array}{ll}\text { Summary } & 32\end{array}$

$\begin{array}{ll}\text { Research Questions } & 34\end{array}$

CHAPTER III: METHODS

$\begin{array}{ll}\text { Theoretical Framework } & 35\end{array}$

$\begin{array}{ll}\text { Raciolinguistics } & 36\end{array}$

$\begin{array}{ll}\text { Research Design } & 38\end{array}$

$\begin{array}{ll}\text { Research Questions } & 38\end{array}$

Mixed Method Concurrent Design 38

Selection of Participants $\quad 41$

Participant Demographics $\quad 43$

Data Collection and Data Sources 46

$\begin{array}{ll}\text { Data Analysis } & 50\end{array}$

Research Questions

$\begin{array}{lr}\text { Summary } & 60\end{array}$

CHAPTER IV: FINDINGS $\quad 61$

Purpose of the Study $\quad 61$

$\begin{array}{ll}\text { Research Questions } & 61\end{array}$

$\begin{array}{ll}\text { Perception Survey } & 61\end{array}$

$\begin{array}{ll}\text { Administrators } & 69\end{array}$

$\begin{array}{ll}\text { Support Personnel } & 69\end{array}$ 
Educators' Perceptions of AAVE as a Legitimate Dialect for Use in an Academic Setting

Administrators

Teachers

Educators' Evaluation of AAVE versus MAE Texts Related to Special Education Referral

Vignettes Written in AAVE

Vignettes Written in MAE

Response to Vignettes by Professional Role

Administrators

Support Personnel

Teachers

Other

Reason for Special Education Referrals

Additional Information Needed

General Educational Factors

Perspectives on Diversity

Special Education Process

CHAPTER V: DISCUSSION

Overview of the Study

Interpretation of Survey Results 
Interpretation of Written Vignettes Results $\quad 86$

$\begin{array}{ll}\text { Perspectives on Diversity } & 88\end{array}$

$\begin{array}{ll}\text { Special Education Process } & 89\end{array}$

$\begin{array}{ll}\text { Limitations } & 92\end{array}$

$\begin{array}{ll}\text { Future Research } & 93\end{array}$

$\begin{array}{ll}\text { Conclusion } & 95\end{array}$

$\begin{array}{ll}\text { REFERENCES } & 97\end{array}$

APPENDIX A: RECRUITMENT LETTER 110

APPENDIX B: INFORMED CONSENT DEBRIEFING 112

$\begin{array}{ll}\text { APPENDIX C: PERCEPTION SURVEY } & 114\end{array}$

APPENDIX D: DEMOGRAPHICS QUESTIONNAIRE 116

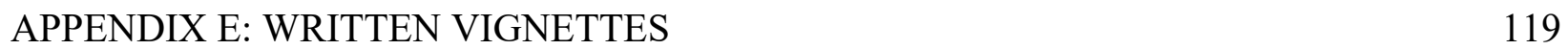




\section{TABLES}

Table Page

1. Definition and Examples of AAVE Selected Morphosyntactic and Phonological Rules 8

2. Educators' Demographic Profiles Response Frequencies and Percentages

3. Codebook Framework and Definitions

4. Educators' Attitudes toward AAVE: Response Percentages and Frequencies

5. Mean Scores and Standard Deviations of Educators' Attitudes towards AAVE by Category

6. Educators' Responses to Vignettes Written in AAVE vs MAE: Response Frequencies and Percentages 


\section{CHAPTER I: INTRODUCTION}

African American Vernacular English (AAVE) is a variation of the Mainstream American English (MAE) dialect spoken in the United States of America (Green, 2002;

Rickford, 1999, 2016). The AAVE dialect has origins steeped within the context of the Atlantic slave trade in the United States and its African American enslaved descendants (Meyer, 2009). Because of this, AAVE has heavy influences from West African languages, General Southern American English, and English-based creoles (Amberg et al., 2009). The AAVE dialect reflects the Niger-Congo languages of African slaves, with paralleling phonetic and grammatical features superimposed with European American English language characteristics spoken by their slave owners (Meyer, 2009).

Approximately $80 \%$ of African Americans in the United States speak AAVE today (Green, 2002). It is a marker of identity and a symbol of racial unity; although not all African Americans speak AAVE, and not all persons who speak AAVE are African American (Rickford et al., 2015). For the purpose of this discussion, the term AAVE speaker refers to African Americans who speak the dialect. Also, in this dissertation, AAVE encompasses all names used over the past four decades to refer to the same dialect, including Ebonics, African American English, Black English, Black Dialect, Afro American English, and African American Language (Mordaunt, 2011). Further, in this manuscript, MAE refers to the dominant English dialect spoken in the United States and encompasses all similar names to refer to it over the years, including Standard American English, Academic English, and Dominant American English (Young et al., 2014).

Although AAVE is one of the most extensively researched dialects in the United States, with decades of researchers substantiating the well-known and widely supported fact that AAVE 
is a complex dialectal language system (e.g., Rickford, 2016; Stockman, Guillory, Seibert, \& Boult, 2013; Van Hofwegen et al., 2010), AAVE is often described as a broken or improper variation of MAE (Gupta, 2010; McKenna, 2013; Newkirk-Turner et al., 2013). It is possible that this is due to a negative perception of the AAVE dialect overall (Gupta, 2010; McKenna, 2013; Newkirk-Turner et al., 2013). Nonetheless, the American Speech Language Hearing Association (ASHA) defines a dialect as a variation of a language system used by a group of people who represent a common region, community, culture, or ethnicity (ASHA, 2018). Further, researchers agree that no single dialect is superior over another one (Godley et al., 2006).

Negative perceptions of AAVE are of particular interest in the field of education within the United States public school system as it relates to disproportionality in special education, which refers to minority students who are over-represented in special education and underrepresented in gifted education (Ford, 2012). For the purposes of this discussion, disproportionality refers to the over-representation of African American students in special education and, therefore, over-identified as having a disability in the public school. Given educators' reported negative perceptions of AAVE overall (Gupta, 2010; McKenna, 2013; Newkirk-Turner et al., 2013), it is possible that AAVE speaking students are mistakenly considered as having a disorder (Bliss et al., 2008; DeJarnette et al., 2015; Rivers et al., 2012). Although teachers generally have students' best interest in mind when making a referral for special education services, conclusions based on subjective judgements may lead to misinterpretation of cultural differences as symptoms of a disability (Herzik, 2015). 


\section{Misperception of Cultural Linguistic Difference as a Disorder}

Labov (1969b) encouraged teachers to learn to distinguish between dialectal differences in AAVE and MAE. This is an important skill to develop so that educators do not mistake AAVE dialectal differences as errors. Labov (1969b) warned educators that failure to recognize the significance and validity of AAVE could lead to inadequate pedagogical solutions of correcting students for linguistic differences and blaming them as though they have errored speech (Rickford, 2016).

Indeed, teachers have consistently expressed their beliefs that AAVE is an inappropriate dialect and that it is infeasible for use at school (Blake et al., 2003; Gupta, 2010). Historically, teachers even dismissed African American children's well-documented AAVE language skills as incoherent (Gay, 2002). Educators' misunderstandings of AAVE can inhibit children's academic success and sense of belonging by inappropriately judging students' abilities based on perceptions about the way they speak. This disapproving attitude can create low expectations of AAVE speaking students as well as inequitable learning opportunities for African American students as a whole (Beneke et al., 2015).

Misperceptions of children's dialects lead teachers to attempt to correct and replace AAVE with MAE. However, researchers demonstrate that correction is not effective when teaching MAE to AAVE speakers (Rickford, 2016). In fact, teachers who use an approach of over-correcting AAVE speakers when teaching MAE risk creating an atmosphere where AAVE speaking students reject school altogether due to a fear of being wrong. This, in turn, disconnects students from the learning experience (Mordaunt, 2011). Further, when children are repeatedly corrected for speaking AAVE, they become self-conscious and overly edit their 
speech. This makes it difficult for AAVE speakers to participate in classroom learning activities equitably (Rickford et al., 2015; Delpit, 2006, 2012).

In all, viewing AAVE as a linguistic impediment may lower teachers' expectations of students who speak the dialect and impact academic outcomes. Also, it may increase feelings of inferiority amongst AAVE speakers. Moreover, this type of deficit thinking may lead to a misdiagnosis of students' ability and possibly to inappropriate educational programming for AAVE speakers. Eventually, this may even lead to AAVE speakers disengaging from school and the learning process altogether (Beneke et al., 2015).

\section{Misperception of AAVE as a Disorder}

With that said, it is possible that AAVE speaking students who use language behaviors different than MAE are mistakenly considered as having a disorder (Bliss et al., 2008; DeJarnette et al., 2015; Rivers et al., 2012). Based on this, educators may need help to identify their own cultural bias so they can properly view their student's behaviors as differences instead of deficits, and consider manners to implement these differences using a method that may improve student learning. When a student's cultural norms differ from the mainstream, it is important to recognize that not all differences are deficits. Instead, it may be crucially necessary to provide assessments, as well as instruction, within the context of the student's cultural linguistic norms (McKenna, 2013).

Researchers have called for teacher education programs to include coursework in linguistic differences to prepare teachers with knowledge to teach children from diverse language backgrounds, including the linguistic properties of AAVE education (Reaser et al., 2008).

Further, researchers proclaim that in order for an educator to plan pedagogical strategies to teach AAVE speakers to read, write, and maximize their oral skills, information on the AAVE 
language system students bring with them is beneficial. Misdiagnosing students' language, social, and cognitive abilities based on a deficit perspective of AAVE has potential to continually perpetuate poor school performance for African American students and over-referral to special education (Reaser et al., 2008). Indeed, there is a gap in the research on effective teaching methods for those who speak the dialect AAVE (United States Department of Education, 2016).

\section{Description of AAVE Instruction Parallel to United States Public School Norms}

It is important to understand and respond to the impact of MAE use on AAVE speakers in schools in the wake of the ever-changing demographics in the United States. According to the National Center for Education Statistics (2016), the population of students from minority racial backgrounds has doubled over the last two decades. African American and Hispanic students now comprise $41 \%$ of the United States combined school population, with African Americans making up $16 \%$ of the United States public school population alone. Nationwide, minority students are projected to represent the numerical majority (55\%) of the school population by the fall of 2026 (NCES, 2016). Meanwhile, the most recent report on racial diversity in the educator workforce indicates that $82 \%$ of teachers are White (United States Department of Education, 2016).

\section{Cultural Linguistic Mismatch}

There is a clear cultural linguistic mismatch in the United States public schools today given the predominantly White teaching force and the increasing number of African American students in school (United States Department of Education, 2016). This information is compounded by the knowledge that approximately $80 \%$ of all African Americans in the United States speak AAVE (Green, 2002). 
It is important to remember that culture and language are intricately intertwined. With culture defined as the values and systems that guide the daily practices of a group of people who have a shared history (Lustig et al., 2012), the very practices found in culture are demonstrated through language practices (Rivers et al., 2012). Further, culture regulates the very interpretation of communication and the context within which they occur, both verbally and nonverbally (DeJarnette et al., 2015). With that said, cultural linguistics is defined as the feelings, opinions, and expectations that groups of people have about how their own group and other groups use language (Hyter et al., 2015).

\section{Solutions to Cultural Linguistic Mismatch}

Given these factors, there remains a need to ensure equitable and valuable school experiences for all Pre-Kindergarten through 12th graders regardless of race, ethnicity, or linguistic expression. In fulfilling this need, educators can become cognizant of responding to students' languages and cultures and the contexts in which they are situated. For instance, when students speak with the AAVE dialect, and are in the process of developing proficiency in MAE, educators can respond by learning about communication patterns associated with AAVE and developing competencies to promote students' navigation within and between dialects (Fallon et al., 2012). This process may increase the students' metalinguistic skills with the two dialects (Terry, 2014), and it may also improve the educators' metalinguistic awareness of the two dialects as well (Craig et al., 2014).

Although linguistic researchers repeatedly establish that AAVE is a legitimate rule-based dialect that is not indicative of a language deficiency, and that it is not indicative of haphazard language skills (Green, 2002; Rickford, 1999, 2016), AAVE continues to be viewed negatively by teachers in the United States (Gupta, 2010; Newkirk-Turner et al., 2014). Also, even though 
researchers show that no one dialect is superior (Godley et al., 2006), students in the United States who speak MAE are held in the highest regard and are generally considered the standard norm within academic settings (Beneke et al., 2015; Ferguson, 2007). Also, it is significant to note that MAE speaking students are most commonly White (Craig et al., 2014).

\section{Linguistic Properties of AAVE}

Linguists often disagree as to whether AAVE is a dialect of MAE or a language of its

own. In fact, the principal professional organization of linguists, called The Linguistic Society of America, highlighted that decisions about distinguishing a language from a dialect are often more social and political than linguistic (Rickford, 2019). For example, linguists commonly view the different variations of the Chinese language as dialects, even though the speakers are completely unable to understand each other. At the same time, linguists commonly view the Swedish and Norwegian vernaculars as different languages, not dialects, even though the speakers are able to understand each other (Rickford, 2019).

AAVE shares many features with MAE; however, it has its own distinct pronunciation and grammatical features (Green, 2002, 2003; Rickford, 1999, 2016; Terry et al., 2016; Wolfram, 2004). Ample research exists acknowledging AAVE as an extensive linguistic system (Green, 2002, 2003; Labov, 1969b) that includes systematic rule patterns in phonology, syntax, semantics, and pragmatics (e.g., Hyter et al., 2015; Newkirk-Turner et al., 2014; Oetting et al., 2010; Rickford, 1999, 2016; Roy et al., 2013; Terry et al., 2016). In other words, AAVE speakers know a unique system of sounds, words, sentence structures, vocabulary meanings, and vocabulary structures, among other information about language (Green, 2002, 2003). Table 1 provides select examples and definitions of phonological and morpho-syntactic rules of the AAVE dialect. 
Table 1

Definition and Examples of Selected AAVE Morphosyntactic and Phonological Rules

\begin{tabular}{|c|c|c|c|}
\hline & Definition & AAVE Example & MAE Translation \\
\hline Zero Copula & $\begin{array}{l}\text { Verb to be omission marks } \\
\text { a single occurrence of an } \\
\text { event as in right now }\end{array}$ & $\begin{array}{l}\text { They waking up too } \\
\text { early. }\end{array}$ & $\begin{array}{l}\text { They are waking up } \\
\text { too early right now. }\end{array}$ \\
\hline Habitual Be & $\begin{array}{l}\text { Verb be marks recurrence } \\
\text { of an event as in all the } \\
\text { time or always }\end{array}$ & $\begin{array}{l}\text { They be waking up } \\
\text { too early. }\end{array}$ & $\begin{array}{l}\text { They always wake } \\
\text { up too early. }\end{array}$ \\
\hline Aspectual Been & $\begin{array}{l}\text { Verb been marks initiation } \\
\text { of an event as in for a long } \\
\text { time }\end{array}$ & $\begin{array}{l}\text { They been waking } \\
\text { up too early. }\end{array}$ & $\begin{array}{l}\text { They have been } \\
\text { waking up too early } \\
\text { for a long time. }\end{array}$ \\
\hline $\begin{array}{l}\text { Initial Voiced th } \\
\text { Sounds }\end{array}$ & $\begin{array}{l}\text { Substitution of } / d / \text { for } \\
\text { voiced /ð/ sound at the } \\
\text { beginning of words }\end{array}$ & /d3m/ & them \\
\hline $\begin{array}{l}\text { Final Voiceless th } \\
\text { Sounds }\end{array}$ & $\begin{array}{l}\text { Substitution of }|f| \text { for } \\
\text { voiceless } / \Theta / \text { sound at the } \\
\text { end of words }\end{array}$ & /bof/ & both \\
\hline $\begin{array}{l}\text { Deletion in Final } \\
\text { Consonant Clusters }\end{array}$ & $\begin{array}{l}\text { Deletion of the second } \\
\text { consonant in final } \\
\text { consonant cluster at the } \\
\text { ends of words }\end{array}$ & /d3s/ & desk \\
\hline $\begin{array}{l}\text { Deletion of } \\
\text { Unstressed Syllable }\end{array}$ & $\begin{array}{l}\text { Deletion of an unstressed } \\
\text { syllable in a multisyllabic } \\
\text { word }\end{array}$ & /prabli/ & probably \\
\hline
\end{tabular}

Note. $\mathrm{AAVE}=$ African American Vernacular English; MAE = Mainstream American English. Definitions and examples are based on the work of Green $(2002,2003)$.

\section{The Role of Codeswitching}

The ability to consciously shift between dialects within language is a pragmatic language skill called codeswitching (Craig et al., 2009; Renn, 2010). Children who speak AAVE speak the dialect considerably more often in informal situations than in formal ones. In formal situations, AAVE speakers typically codeswitch to MAE. In fact, African American children speak AAVE almost twice as often in informal settings with their peers than in formal ones. In other words, 
African Americans who speak AAVE present with intuitive cognizance of the phenomenon of codeswitching, as they naturally respond to functions of situational circumstances in modifying their use of the dialectical features in specific contexts (Renn, 2010).

\section{AAVE, Codeswitching, and Education}

Researchers have long speculated that students who codeswitch from AAVE to MAE are impacted in their ability to achieve academically in literacy skills (e.g., Labov,1967; Wolfram, 2004). Indeed, researchers found that when children were able to codeswitch from AAVE to MAE at school, it influenced academic reading achievement test scores in verbal language and writing. In fact, test scores in reading achievement are inversely connected to AAVE production, with success declining considerably between both oral and written narratives when students used AAVE during reading tasks. In other words, as AAVE speakers use MAE during literacy tasks, they perform better in reading achievement than their peers who do not use MAE (Craig et al., 2009; Craig et al., 2014; Terry et al., 2016; Terry et al., 2012). Even when comparing African Americans along those of similar and different social economic status, use of AAVE, specifically, the inability to codeswitch from the AAVE to MAE, was a greater indicator of performance on language and literacy tasks, than social economic status (Gatlin, et al., 2016).

While AAVE speakers show a natural disposition towards codeswitching to MAE (Renn, 2010) and present with improved academic achievement when codeswitching between AAVE and MAE (Craig et al., 2009), promoting a conscious shift towards codeswitching from AAVE to MAE is not always embraced in the classroom setting (Creese et al., 2010), possibly due to a negative perception of the dialect (Gupta, 2010). However, educators can help young children acquire knowledge of an all-inclusive language system through repeated exposure to communication patterns in different contexts (Clark, 2007), allowing AAVE speakers to develop 
linguistic flexibility through codeswitching. In other words, encouraging African American students to learn how to switch between the language expectations of AAVE at home and MAE at school through use of both AAVE and MAE at school can effectively empower students to make choices about how they use language in different settings (Brown 2006; Dyson et al., 2009). In this way, teachers can support AAVE speakers in their linguistic experiences while simultaneously teaching explicit MAE language and literacy skills (Clark, 2007).

Indeed, AAVE speakers who learn to bi-dialectally speak MAE are more successful academically than their peers who do not learn to code-switch between AAVE and MAE (Craig et al., 2009; Renn, 2010; Renn et al., 2009). When discussing codeswitching, it is helpful to view teaching MAE to AAVE speakers as becoming proficient in two dialects, or becoming bidialectal. This is not to be confused with remediation, which is a negative practice within the cultural linguistic context of codeswitching (Mordaunt, 2011). As the principles of Vygotsky's (1978) sociocultural theory states, students bring their own valuable knowledge to the learning environment. Therefore, condemning a students' home language or culture only serves to disconnect them from the learning environment at school, which is charged with building students with additional knowledge. Instead, teaching codeswitching while honoring dialectal awareness boosts students' self-confidence, and enhances their relationship with teachers and school in general. As educators learn about AAVE and recognize its legitimacy as a rulegoverned language system, and a valid dialect of MAE, their perspective toward speakers of the dialect will naturally change (Campbell et al., 2017; Mordaunt, 2011).

\section{Remedies to School Norms Disruption}

Because schools are becoming progressively more diverse, it is important for teachers to embrace cultural diversity. This includes knowledge about the unique characteristics, 
knowledge, and histories of each given group of people, including African Americans and the AAVE dialect. It is true that each student benefits when they receive maximum value from their school experience, and when their culture and context are adequately considered. For this reason, it is essential to embrace linguistic diversity as a strength, and build upon educators' abilities to understand CLD cultures, including the African American culture, through recognition of the extensive dialectal language system of AAVE (Fallon et al., 2012; Lobeck, 2019).

\section{Culturally Relevant Pedagogy}

Ladson-Billings' (1995) approach to educating African American students is a seminal work on culturally relevant teaching. Ladson-Billings' work focuses on developing teachers that effectively use critical pedagogy that specifically empowered African American students. Culturally relevant pedagogy specifies the use of students' linguistic culture when providing intellectual, social, emotional, and political instruction. This includes CLD students' unique, meaningful experiences within the learning process, acquisition of knowledge, and perspective attitudes (Ladson-Billings, 1995). Culturally relevant pedagogy demands student success through teacher development of cultural competence, critical consciousness, and challenges against the status quo (Harmon, 2012). Specifically, culturally relevant pedagogy teaches educators to build bridges, or scaffolds, from African American students' knowledge to experiences at school to facilitate achievement and academic success (Ladson-Billings, 1995, 2000, 2006).

\section{Cultural Competence}

Cultural competence is a model of culturally relevant pedagogy used by many educators. This framework teaches educators to be self-aware about the languages, dialects, and cultures of others, as well as their own. Culturally competent educators are defined as ones who 
simultaneously appreciate cultural and linguistic differences, and actively participate in selfanalysis to assess cultural biases and improve self-awareness (ASHA, 2018). Culturally competent educators utilize research-based practices that take into account the communication backgrounds and needs of the students and their families by studying disorders within a social framework related to contexts relevant to the student (ASHA, 2018). This is a critical skill when evaluating students who speak AAVE, so that conclusions regarding disorder versus dialectal differences are not based on standardized norms that may be irrelevant to the student (Beneke et al., 2015; Craig et al., 2014; Oetting, 2018).

\section{Culturally Responsive Pedagogy}

Cazden (1981) first used the term culturally responsive to describe interactions between White American teachers and Native American students who spoke different languages and came from different cultures. Gay $(2002,2010)$ used the term to further describe teaching CLD learners as a whole through education that was multidimensional, empowering, and transformative. To date, culturally responsive pedagogy is named by leaders in the field as one of the most effective instructional tools designed to meet the learning needs of CLD students, including African Americans who speak AAVE (Gay, 2002, 2010; Harmon, 2012; LadsonBillings, 1995, 2000, 2006).

Culturally and linguistically responsive pedagogy with AAVE speaking students can build on their knowledge and experiences to successfully transform the typical curriculum to one that facilitates achievement and academic success. Specifically, instruction successfully sets high expectations for African American students and provides progressive attentiveness to nurture academic success. It honors African American students' cultural linguistic knowledge of AAVE and employs families as a resource to grow and maintain students' cultural competence. Also, 
culturally responsive pedagogy involves students in exploring challenges within their own communities to develop analytical cognizance (Irvine, 1989, 2002; Ladson-Billings, 1995, 2000, 2006).

Not only does culturally and linguistically responsive pedagogy include transformative teaching through incorporation of a multicultural content model, but it also implores social action through social justice amongst educators and students alike. A multicultural model allows for voices which have been historically excluded, like those of AAVE speakers, to express opinions, values, and beliefs. This allows AAVE speaking students to explore ways to impact change within society. Further, it aids teachers in addressing the inequities within the United States public system (Harmon, 2012).

Lastly, the culturally and linguistically responsive pedagogy builds upon cultural assetbased instruction, using cultural assets as the foundation for instruction. Particularly, cultural assets refer to cultural pragmatic behaviors that African American students bring to the classroom which may be viewed as challenging to educators who are not knowledgeable about African American culture. This is especially true of teachers who subscribe to deficit thinking. It is important to note that cultural assets include student development of cultural identity, which, in terms of AAVE, includes communication, individualism, emotion, and social point of view (Harmon, 2012).

\section{Statement of the Problem}

\section{Disproportionality in Special Education}

Historically, large numbers of African Americans students have been inappropriately placed in special education settings because their academic knowledge, primary language, or language dialect differed from those that were typically used in the standard school setting 
(Delpit, 2006). When teachers make referrals to special education, they often use their own perception of a problem to drive the initial process. Frequently, African American students are referred for evaluation by special education team members who apply a deficit lens to interpreting language behavior patterns as indicators of a disabling condition (Blanchett, 2006; Bliss et al., 2008; DeJarnette et al., 2015; Harry et al., 2014; Hwa-Froelich et al., 2007; Rivers et al., 2012). Since the great majority of students who are referred for a special education evaluation are subsequently placed in special education, examining the referral process itself may be an important first step in addressing disproportionality in special education (Dever et al., 2016; Linton, 2014).

Misperception of African American culture may be leading to African American students being disproportionately over-represented in special education (Blanchett 2006; Skiba et al., 2008). Based on discrepancies between identification of disabilities with subjective descriptions and objective descriptions, educators' misperceptions of linguistic and cultural differences found in the AAVE dialect may well be playing a role in inappropriate referrals for special education and a subsequent misdiagnosis of a disability (Rickford, 2016).

\section{Policy Related to Disproportionality}

In 2004, Congress acknowledged the issue of disproportionality in the Individuals with Disabilities Education Act (IDEA), reiterating the need to address the overrepresentation of minority students in special education, including African American students (20 USC. 1416(a)(3)(C). In 2012, the Council for Children with Behavioral Disorders (CCBD) openly recognized the magnitude of racial disproportionality in special education after Congress labeled it one of their top areas of concerns in IDEA 2004 (CCBD, 2013). In 2016, the United States Department of Education issued regulations termed Equity in IDEA for significant 
disproportionality which brought changes to how states and school districts would be required to monitor, analyze, and report special education practices and policies that address disproportionality in special education $(34 C F R \S 300.646,2016)$.

\section{Purpose of the Study}

The purpose of this study is to investigate the special education referral process for African Americans who communicate using AAVE in written form as researchers have long speculated that students who codeswitch from AAVE to MAE are impacted in their ability to achieve academically in literacy (e.g., Labov,1967; Wolfram, 2004). This was completed to gain a better understanding of the causes of disproportionality in special education. Further, through this study, I hope to gain insight into the perceptions of special educator personnel of students who AAVE and its perceived impact on disproportionality in special education. Also, this study will provide information about educators' training and use of cultural linguistic practices for AAVE speaking students in the classroom setting.

For the purposes of this study, MAE is defined as the mainstream dialect of American English used primarily at school, and AAVE is defined as a linguistically rule-based dialectal variation of MAE spoken by many, but not all, African Americans. Findings from this study could potentially provide effective ways for constructing university education courses to improve educators' knowledge of the African American culture, the significance of the rule-based system of AAVE, and awareness of its impact on disproportionality in special education. Also, this study may be useful in incorporating with professional development training sessions for inservice educators and education related personnel in the future (e.g., speech-language pathologists, psychologists). Stakeholders include researchers studying teacher preparation 
programs, teacher educators who are interested in cultural and linguistic diversity, education professionals, and curriculum developers for culturally responsive pedagogical practices.

\section{Significance of the Study}

It is possible that African American children and adolescents may display language behaviors that are culturally different than their White peers, and may erroneously be identified as having a disability (DeJarnette et al., 2015; Rivers et al., 2012). Knowledge and understanding of African American culture and linguistic difference in AAVE is particularly important for education personnel to be aware of within the special education referral process (DeJarnette et al., 2015; Rivers et al., 2012). A misunderstanding of the linguistic differences found in AAVE spoken by many African American children and adolescents paired with educators' misperception of the dialect, may be contributing to the over-referral and over-identification of African American students as disabled (Hyter, Rivers, DeJarnette, 2015; Rivers et al., 2012).

\section{Positionality}

I am a speech-language pathologist with 20 years of experience in urban education and healthcare settings with children and adults ages birth through geriatric. I hold active speechlanguage pathology certification with the American Speech-Language-Hearing Association (ASHA); an active Professional Educator License through the Illinois State Board of Education with an endorsement in speech-language pathology for pre-kindergarten through age 21; an active Director of Special Education License through the Illinois State Board of Education; and an active state license through the Illinois Department of Financial and Professional Regulations. I hold a Master of Arts degree in speech-language pathology and am currently working towards a Doctoral Degree in Special Education at a state school. I am an African American female and 45 years of age. I communicate using both AAVE and MAE. 
I also serve as a leader in special education advocacy through work as the Professional Development Chair for the Division for Culturally and Linguistically Diverse Learners (DDEL) at the Council for Exceptional Children (CEC). Over the past three years, I have served in various capacities through CEC DDEL, including Governmental Liaison and Vice President, and actively worked to persuade stakeholders in the Office of Special Education Programs (OSEP) at the US Department of Education for fair treatment, equal rights, and equity in education for African American students. My primary research interests encompass the validation of AAVE in the wake of the nation's ongoing conversations about implicit racial bias. As a leader in education, I strive to stir educators' knowledge about the difference between a dialect and a disorder, raise awareness about the possibility of its connection to disproportionality in special education, and embolden educators to change their perspectives and actions towards those who speak AAVE based on these truths. I am particularly interested in the over-referral and overidentification of African American students as having emotional behavioral disorders and specific learning disabilities in children and adolescents in the African American culture.

As an African American who speaks both AAVE and MAE, I present with a bias towards equal value of both dialects. Further, my bias extends to assume that participants do not hold equal value of both MAE and AAVE and that referral decisions are based on devaluing of AAVE by participants. To minimize bias, I will check for alternative explanations and verify with additional data sources. Alternative explanations will be addressed through asking participants to explain why they made a decision to refer the $3^{\text {rd }}$ grade to special education. Verify with additional data source will be addressed through use of two data collection elicitations for the same dialectal vignette style of MAE and AAVE in addition to a survey to assess overall perceptions of AAVE. 


\section{Summary}

Exploring and addressing teaching practices and student outcomes as it relates to cultural linguistic differences in AAVE may help propel the knowledge of culture and cultural competence within the field of education. This will urge educators to use their own knowledge of the complex view of culture and language to make decisions about student performance, appropriate interventions, and referrals to special education. It may also assist educators in developing a willingness to explore and address insights into language and culture using a systematic and interdisciplinary approach. Also, improving understanding of inclusive education practices, providing educational system accountability, and offering opportunities for all students to learn may even serve to strengthen core instruction for AAVE speakers (Artiles, 2015).

Ideas of building bridges between home and school culture suggests that educators provide support for diverse dialects like AAVE. This includes educators recognizing the importance of reflecting on teachers' own culture and dialect as well as honoring diverse English dialects of students and families (Artiles et al., 2007; Artiles et al., 2011; Ladson-Billings, 2006). Ingrained dominant-focused educational practices, however, continue to be a barrier to equity and inclusion as United States public school systems are designed to respond to student-family deficits rather than student-family strengths (Artiles et al., 2007; Artiles et al., 2011). To that end, a revised set of practices may advance educational outcomes for students like AAVE speakers who are most vulnerable. In all, greater equity and inclusion for children who speak AAVE would provide a purpose for educators to understand dialect characteristics and their importance. Deep-seated practices can be overturned to support greater access to learning as well as inclusion of all CLD marginalized people groups, including African Americans. 
Moreover, inclusive practices hold potential for avoiding inappropriate referral and placement of AAVE speaking students in special education (Beneke et al., 2015). 


\section{CHAPTER II: LITERATURE REVIEW}

This chapter introduces literature pertaining to disproportionality in special education and its possible connection to African Americans who communicate using AAVE. Concerns about the possibility of educators' misperception of AAVE as a disorder are discussed. Based on these concerns, a literature review was conducted to gather empirical peer-reviewed studies associated with United States public schools between 2004 - 2019 in response to Congress' charge to address significant disproportionality in IDEA 2004.

\section{A Review of the Literature}

Based on concerns about disproportionality in special education and a gap in the knowledge and understanding of teachers' perceptions of AAVE speakers and its impact on the special education referral process, this literature review was conducted to gather empirical peerreviewed studies associated with United States public schools on these issues. The timeframe for this literature review was set to $2004-2019$ in response to Congress' charge to address significant disproportionality in IDEA 2004.

A search for peer-reviewed journal articles occurred over seven electronic databases. Due to a limited amount of studies regarding educators' perception of AAVE, special education referrals related to AAVE, and AAVE-related research based practices, initial searches broadly included qualitative, quantitative, mixed methods, practitioner articles, and dissertations (Hyter et al., 2015). Databases included Google Scholar; PsychINFO; all Eric Resources Information Center databases; Complementary Index; Education Full Text; Academic Search Complete; Social Sciences Citation Index; and Arts and Humanities Citation Index. A hand search was also completed to review journals, including American Journal of Speech-Language Pathology; Language, Speech, and Pragmatic Language of African American Children Hearing Services in 
Schools; Journal of Speech, Language, and Hearing Research; and Communication Disorders Quarterly. Multiple key words guided the search in various combinations both individually and in groupings based on a generalized theoretical framework related to AAVE, educators' perception, and culturally responsive practices. Key words included: belief, perception, attitude, bias, African American English, African American Vernacular English, African Language, Black English, Ebonics, referral, special education, and disproportionality.

In the search for peer-reviewed journal articles, the cutoff number for African American student intervention participants was set at $30 \%$ to include research studies containing other minority groups of students. The cutoff number for general education teachers, special education teachers and/or related service personnel was set at $30 \%$ of the participants to exclude studies that relied primarily on self-assessment or self-intervention tools using K-12 students' and/or their parents'/guardians' self-report to reach conclusions. Articles published in a language other than English or not occurring in the United States were also excluded, as well as book chapters, conference presentations, dissertations, and master's theses. To ascertain which articles were relevant, abstracts of each were read and only articles that (a) included African American students as at least $30 \%$ of the participants for intervention in a study designed to address AAVE speakers compared to their MAE peers, (b) included pre- or in-service general education teachers, special education teachers and/or related service personnel as at least $30 \%$ of the participants for school educators in a study designed to address AAVE speakers compared to their MAE peers, and (c) were conducted after 2004, were included.

Initial results yielded a total of 63 articles; however, after removing articles based on predetermined exclusionary characteristics of empirical studies related to general education teachers, special education teachers and/or related service personnel's perceptions of AAVE 
speakers and/or use of culturally responsive teaching strategies to assist AAVE speaking students with learning MAE, only 24 articles remained. Additionally, after excluding articles not from peer reviewed journals, only seven articles remained

Results of the literature revealed a limited amount of empirical research studies focusing on educators' perception of AAVE after receiving instruction on the rule-based system and the special education referral process for African American students. Seven articles were identified in total. The articles were coded by key themes when more than one article represented the same theme. When only one article was identified within a theme, the article was coded as a key idea. Of the seven articles identified in this literature review, there was overlap in identified themes, with some articles highlighting more than one theme. In all, seven studies reviewed (Blackburn, 2012; Champion et al., 2012; Fogel et al., 2006; Gupta, 2010; Newkirk-Turner et al., 2013; Shepherd, 2011; Skiba, Simmons, et al., 2006) yielded two primary themes: (a) perceptions of AAVE and (b) over-referral of African American students to special education. There was only one article within the specific categorical theme of over-referral of African American students to special education related specifically to African American cultural linguistic practices (Skiba, Simmons, et al., 2006), and there were six articles highlighted within the categorical theme of educators' perceptions of AAVE (Blackburn, 2012; Champion et al., 2012; Fogel et al., 2006; Gupta, 2010; Newkirk-Turner et al., 2013; Shepherd, 2011). Of the six articles related to perceptions of AAVE, there were three overlapping themes across articles: (a) four articles highlighted themes related to educators' overall perception of AAVE speakers (Champion et al., 2012; Gupta, 2010; Newkirk-Turner et al., 2013; Shepherd, 2011), (b) three articles highlighted themes of educators' perceptions of preparedness to address the needs of AAVE speakers (Champion et al., 2012; Gupta, 2010; Newkirk-Turner et al., 2013), and (c) two 
articles highlighted themes of educator training on AAVE and its impact on perception (Blackburn, 2012; Fogel et al., 2006).

\section{Perception of AAVE}

\section{Educators' Overall Perception of AAVE}

Gupta (2010) and Shepherd (2011) conducted surveys of 156 and 57 in-service elementary teachers, respectively, with the purpose of examining their attitudes towards the AAVE dialect. Gupta (2010) examined the attitudes of 156 in-service teachers towards AAVE, including 20 special education teachers, all elementary district with selected public schools. Results indicated belief that AAVE was an inadequate language system (86\%), AAVE contributed to problems with academic success in reading (59\%), writing (73\%), and performance in language arts (78\%). Respondents reported receiving inadequate preparation to address linguistic needs of speakers of AAVE (67\%), that addressing linguistic needs of AAVE speakers would promote academic achievement (70\%), and that they would like to learn strategies to address the linguistic needs of students who speak AAVE (70\%). Implications were that here is need for teacher in-service training and pre-service teacher preparation programs regarding culturally responsive practices for AAVE speakers. Recommendations included requiring pre-teacher courses that includes a heavy emphasis on language that provides a foundation to address minority differences in the classroom.

Shepherd (2011) evaluated 57 in-service teachers' perception of pre-recorded speech spoken by White and minority second and third grade students without the aid of a visual. The in-service teachers were enrolled in a master's level teaching program and included White (57\%), Hispanic (30\%), and African American (7\%) respondents. The participants listened to

pre-recorded identical sentences delivered by minority and White boys and girls in second and 
third grade. Verbal responses made by minority boys, minority girls, and White boys were evaluated less favorably than those by White girls. In-service teachers rated perceptions of minority boys' speech less favorably than they did White boys and minority girls with an average of 0.14 standard deviations less favorably for minority boys, 0.16 standard deviations less favorably for White boys, and 0.18 standard deviations less favorably for minority girls. On average, Black and Hispanic teachers perceived speech from minority boys and girls less favorably than from White girls. Further, Shepherd (2011) employed a linear regression confirmed that Black and Hispanic teachers perceived speech from minority boys and girls less favorably $(\beta=.091, \mathrm{t}(680)=2.31, \mathrm{p}<.025)$ than White and Asian teachers $(\beta=.091, \mathrm{t}(680)=$ $1.98, \mathrm{p}<.05)$. Implications and recommendations included the need to make teachers aware of their perceptions and expectations of students of different minority groups to guard against bias that could impact student learning.

In all, results of the in-service teacher surveys revealed that a majority of in-service teachers perceived AAVE as an inadequate language system (Gupta, 2010) that was less favorable than MAE (Shepherd, 2011). In fact, in-service teachers directly attributed the dialect as contributors to problems with academic success amongst African American students. Most reported perceived negative impact on reading and writing skills and language arts (Gupta, 2010). Further, amongst in-service teachers, increased negative perceptions occurred towards AAVE speaking boys rather than AAVE speaking girls (Shepherd, 2011).

Similarly, pre-service teachers also reported negative perceptions about AAVE (Champion et al., 2012; Newkirk-Turner et al., 2013). Champion et al. (2012) examined general attitudes towards AAVE and perceptions of AAVE as a legitimate dialect that should be used in the classroom setting amongst 136 preservice teachers in two sections of an undergraduate 
required course titled 'Teaching Diverse Population', White (77\%), African American (9\%), Hispanic (4\%). Results indicated negative views about AAVE and inadequate training in language diversity, including AAVE (85\%). White students expressed negative perceptions of AAVE while African American and Hispanic students expressed more favorable opinions about the dialect. Implications were that pre-service teachers should receive training on different dialects of English that minority students may speak at school. Recommendations included the consideration of best practices, such as appropriate evaluation of dialectal speakers before rushing to judgement about impairment.

Newkirk-Turner et al. (2013) examined the attitudes of 38 pre-service undergraduate teachers in elementary schools of a selected public school district, majority African-American respondents (90\%), at Historically Black College and University Jackson State University teachers towards AAVE. Results indicated negative perception of AAVE as inadequate language system (61\%), adverse impact on educational outcomes including writing $(61 \%)$ and performance in language arts (71\%), feelings of unpreparedness to address the needs of AAVE speakers in the classroom and need for additional strategies (82\%). Implications included the need for a systematic focus on culturally responsive practices for AAVE speakers in teacher preparation programs. Recommendations were to implement an interdisciplinary approach to teacher preparation that includes SLPs providing trainings on linguistic diversity.

In all, via a Likert-style survey, 136 (Champion et al., 2012) and 38 (Newkirk-Turner et al., 2013) pre-service teachers participated in a study with the purpose of examining attitudes towards AAVE. Results of these studies revealed feelings that students who spoke AAVE would experience adverse impacts on educational outcomes, with $61 \%$ of pre-teachers perceiving negative impacts on writing and $71 \%$ perceiving negative impacts on language arts (Newkirk- 
Turner et al., 2013). This highlights teacher report of uncertainty of ways to best address the needs of AAVE speakers (Gupta, 2010; Newkirk-Turner et al., 2013).

Of the in-service educators who reported perceptions of AAVE, several were in-service teachers who worked in special education (Gupta, 2010), and many of the surveyed pre-service teachers were African American (Newkirk-Turner et al., 2013). This highlights the potential of bias even amongst African Americans and special educators who one might expect to not have preconceived prejudicial notions based on disability or race (Annamma et al., 2013). With that said, when reflecting on an ethnically diverse sample of pre-service teachers overall, White preservice educators expressed negative beliefs about AAVE speaking students at a greater rate than African American pre-service educators (Champion et al., 2012).

\section{Educators’ Perception of Preparedness to Address AAVE Speakers}

In-service and pre-service educators reported feelings of inadequate preparation to address the linguistic needs of AAVE speakers (Gupta, 2010; Champion et al., 2012; NewkirkTurner et al., 2013). In fact, many pre-teachers expressed inadequate training in language diversity outside of MAE altogether (Champion et al., 2012). Likewise, the majority of inservice and pre-service teachers expressed a desire to learn strategies that address the linguistic needs of students who speak AAVE (Gupta, 2010; Newkirk-Turner et al., 2013; Champion et al., 2012). Moreover, $70 \%$ of in-service teachers and $61 \%$ of pre-service teachers indicated beliefs that addressing the linguistic needs of AAVE speakers would help to promote students' academic achievement (Gupta, 2010; Newkirk-Turner et al., 2013).

\section{Educators' Training on AAVE and Impact on Perception}

Two studies between 2004-2019 focused on training educators on the dialectal features of AAVE with measurements of educators' knowledge and perception of the dialect before and 
after the intervention (Blackburn, 2012; Fogel et al., 2006). Blackburn (2012) instructed 63 undergraduate speech-language pathology (SLP) students on seven grammatical rules in AAVE and Fogel and Ehri (2006) trained 73 MAE-speaking teachers on the same seven grammatical features while enrolled in three master's-level teacher education programs. The AAVE syntactic rules included in the study were: (a) omission of plurals (b) omission of regular past tense $-e d$, (c) omission of possessives (d) omission of the copula, (e) omission of third person singular present tense, (f) subject expression, and (g) use of indefinite articles (Blackburn, 2012; Fogel et al., 2006).

Blackburn (2012) examined 61 speech-language pathology students' knowledge of AAVE and attitudes toward AAVE before and after coursework on AAVE and linguistic diversity in a female undergraduate speech-language pathology program from two sections of a required course for select AAVE phonological and grammatical rules listed above. Results indicated that participants significantly improved knowledge of AAVE phonological $(\mathrm{t}(61)=5$. $05)$ and grammatical $(\mathrm{t}(61)=5.05, \mathrm{p}<.0)$ features. Students improved attitudes towards clinical practice with clients who speak AAVE at school (pre-test 78\% negative attitudes towards AAVE, post-test $19 \%$ negative attitudes toward AAVE). Implications were that dialect instruction can improve students' knowledge of non-MAE dialects with recommendations to continue exploring ways to improve SLP trainings to better prepare SLPs for working with minority students in schools.

Fogel et al. (2006) conducted a study with 73 in-service teachers enrolled in three sections of master's level teacher education programs, including demographics of White (78\%), Black (12\%), Hispanic (7\%), and Asian (3\%). The purpose of the research was to study the process of educating MAE speaking teachers on the rules of AAVE: (a) exposure to AAVE plus 
explanation of dialect modification strategies and (b) exposure, strategy explanation, and guided practice translating sentences from MAE to AAVE for select features described above. Results indicated that all teachers improved in knowledge and positive attitudes towards AAVE but teachers who received guided practice were more effective in translating sentences from MAE to AAVE. An increase from $14 \%$ on the pretest to $79 \%$ on the posttest as compared to those who did not receive guided practice with a score of $13 \%$ on the pretest and $44 \%$ on the posttest. Implications were that self-regulated learning theory is effective to teach AAVE and MAE dialectal features to teachers so they can help AAVE speakers learn to code-switch. Recommendations were to implement additional studies that examine ways to teacher educators about AAVE.

Both the Blackburn (2012) and the Fogel et al. (2006) study explored comparisons between the same two instructional learning practices: (a) exposure to AAVE plus explanation of dialect modification strategies and (b) exposure, strategy explanation, and guided practice of written translating sentences from MAE to AAVE. Results revealed that for both intervention groups, educators' attitudes towards AAVE speakers dramatically improved, and that familiarity and understanding of AAVE features increased as demonstrated through written translation tasks (Blackburn, 2012; Fogel et al., 2006). With that said, those who received guided practice instruction improved at greater effects in translation tasks than those who did not. These results suggest that instruction on dialectal differences may improve educators' knowledge and perception of AAVE and that guided practice may encourage translation practices, for all people, from MAE to AAVE, and potentially AAVE to MAE (Blackburn, 2012; Fogel et al., 2006).

Training educators to use culturally responsive pedagogical practices in the classroom is a growing body of research (Blackburn, 2012; Fogel et al., 2006). For example, explicit 
instruction on AAVE leads to accurate written translation of morphosyntactic rules between AAVE and MAE for teachers (Fogel et al., 2006), and it elicited accurate translation amongst speech-language pathologists in graduate training as well. Moreover, translation activities also increased positive views of AAVE amongst speech-language pathologists in training (Blackburn, 2012), in the same way that the training did for teachers in the features of AAVE (Fogel et al., 2006). Moreover, training pre-teachers to use culturally responsive pedagogy in AAVE using service learning in elementary schools has great potential given its success at increasing preteachers' cultural competence regarding language and literacy instruction during on-site elementary school practicum (Endo, 2015).

Further, Fogel and Ehri (2006) found that MAE speaking teachers who practiced translating sentences from MAE to AAVE after explicit instruction and corrective feedback, effectively learned to translate AAVE and MAE during writing tasks. When teachers incorporated their understanding of AAVE linguistic characteristics, they learned strategies that supported AAVE speakers that they could apply in practice within the classroom setting. Moreover, the teachers became more sensitive to the needs and abilities of their students who spoke AAVE and their attitudes towards AAVE positively increased as they became more acquainted with its features (Fogel et al., 2006).

\section{Over-referral of African American Students to Special Education}

Only one study sought to gain insight of the referral process and how it may contribute to the phenomena of disproportionality of African American students in special education. Although this study did not specifically address AAVE speakers in particular, it did address African American students who were economically disadvantaged along with the phenomena of disproportionality and special education referrals. (Skiba, Simmons, et al., 2006); and it is known 
that $80 \%$ of African Americans speak AAVE (Green, 2002). However, there is no explicit connection made from AAVE to disproportionality in special education in this article, although this article directly addresses African Americans. I am making the connection of AAVE and disproportionality in special education to examine the possibility that language difference may be impacting the special education referral process. In fact, no articles were found that specifically address perception of AAVE and the special education referral process (Skiba, Simmons, et al., 2006).

In the study, Skiba, Simmons and colleagues (2006) interviewed 66 educators, including psychologists, special education directors, principals, and classroom teachers about their perceptions on urban education, special education, diversity, disproportionality, and their accessible and desired resources (Skiba, Simmons, et al., 2006). Skiba, Simmons, and colleagues (2006) found that student behavior was the primary driving force within the initial special education referral process for many teachers. Further, the study highlighted that there are gaps in educators' understanding of culture, which may intensify teachers' challenges with perception of student behavior (Skiba, Simmons et al., 2006). This is significant given the intertwining relationship with culture and pragmatic language (DeJarnette et al., 2015).

Within the United States (U.S.) public schools, when educators refer to culturally and linguistically diverse (CLD) learners, what they really mean is culturally and linguistically different - different from the White mainstream norms that are postulated within the United States public school system within the MAE dialect (Seltzer \& Rios, 2018). This is true when considering pragmatic language skills amongst African Americans as well; as they differ from White mainstream norms (Green, 2002). If teachers plan to present learning environments that are inclusive and welcoming to all people, valuing what students bring to the learning 
environment includes valuing dialectal differences like AAVE as well. Understanding and embracing cultural linguistic values allows students to play an active role in their own education, recognizing that interactions between teachers and students are paramount in student achievement (Craig et al., 2009, 2014; Terry et al., 2012, 2016). For this reason, the study by Skiba, Simmons and colleagues (2006) was embraced to examine the possibility of pragmatic language difference and its impact on the special education referral process.

However, with the goal of developing AAVE speakers' acquisition of MAE being to become bidialectal, or linguistically proficient in both AAVE and MAE dialects, then instruction should also foster a learned purpose of promoting self-confidence and self-trust. To do this, it is important that instruction is celebratory of African American students, without perpetuating reverence for White American culture over an African American one (Campbell et al., 2017). Because of this, in addition to the sociocultural theory (Vygotsky, 1978), it may also be beneficial to consider the sociolinguistic perspective as well. The sociolinguistic theory views all dialects as equally sound and grammatically valid and combats negative perceptions of AAVE through active examination of discrimination and prejudice based on language. Further, the sociolinguistic theory allows for open acknowledgement that the MAE dialect operates from a dominant position of social power and capital gain and that negative perceptions of AAVE are based on faulty ideologies (Labov, 2001).

Also, many teachers reported perceptions that special education was the only resource available to meet the needs of students who were not succeeding in school. Further, teachers seemed less concerned about whether or not the special education services students received were effective, and more concerned that the students who needed help, received it. Moreover, teachers were surprisingly reluctant to discuss issues surrounding race and ethnicity with the 
researchers. Overall, these results highlighted the complicated nature of the extensive factors that contribute to and perpetuate disproportionality in special education for African American students (Skiba, Simmons, et al., 2006), including those who speak the dialect AAVE (Green, 2002).

\section{Summary}

The results of this literature review revealed that there are gaps in the research related to perceptions of AAVE and its connection to disproportionality in special education. In all, these reviewed articles affirmed that educators have a negative perception of AAVE speakers (Champion et al., 2012; Gupta, 2010; Newkirk-Turner et al., 2013; Shepherd, 2011), with particular negative feelings towards African American boys (Shepherd, 2011); however, there is a gap in the knowledge of how these negative perceptions impact student education, the special education referral process, and disproportionality in special education.

Both pre- and in-service educators reported wanting additional information on the AAVE dialect and how to address the needs of AAVE speakers in the classroom (Champion et al., 2012; Gupta, 2010; Newkirk-Turner et al., 2013). Further, when in-service and pre-service educators did receive tools to learn more about the linguistic rules of AAVE, their perceptions of the dialect improved, along with their knowledge and awareness of the rule-based system of AAVE. However, there is a gap in the knowledge of educators receiving tools regarding interventions to be used with students who speak AAVE and its impact on educators' perception and understanding of the dialect (Blackburn, 2012; Fogel et al., 2006).

Results of the empirical studies also highlighted that both African American and White educators have a negative perception of AAVE (Newkirk-Turner et al., 2013) but there is a gap in the research knowledge of how this impacts students' educational experience. Further, there is 
a gap in the research regarding educators' perception of the cultural linguistic behavior of AAVE speakers and its influence on the initial special education referral process. Moreover, results indicated that reluctance to having open discussions about race relations illuminates the complicated multifaceted issue of disproportionality in special education.

Also, teachers reported inadequate access to resources that support differentiated instruction for African American students (Skiba, Simmons, et al., 2006). Further, teachers expressed the need for access to materials designed to support primary AAVE speakers (Green, 2002). Results of the literature review suggested that successful remedies to support AAVE speakers and diminish disproportionality in special education in the United States public schools may well include increasing educators' knowledge and understanding of cultural linguistic differences in AAVE speakers (Blackburn, 2012; Fogel et al., 2006). Also, since the initial referral process to special education is largely based on teachers' perceptions about student behavior (Skiba, Simmons, et al., 2006), researchers suggest there is need to develop resources and policies that address teacher understanding of AAVE speakers' cultural and linguistic needs in education (Hyter et al., 2015; Skiba, Simmons, et al, 2006).

In all, results of the literature review revealed that to address the crucial needs of AAVE speakers in a way that might have a realistic impact on disproportionate overrepresentation of African Americans in special education, there is need to address the gap in the research that examines educators' views towards cultural differences in AAVE speakers. Further, there is need to refrain from oversimplifying the multifaceted issue of disproportionality in special education, and embrace difficult conversations surrounding race and perception in the teacher to student relationship to address the gap in research on this issue. Moreover, there is a gap in the research on the full range of language skills of AAVE speakers to provide educators with a cohesive 
catalogue of typically developing African American children and adolescents which may help educators better differentiate between cultural linguistic difference and disorder in African American AAVE speaking students (Hyter et al., 2015; Skiba, Simmons, et al, 2006).

\section{Research Questions}

The research questions for this study were:

1. What are the perceptions of in-service teachers, support personnel, and/or administrators towards the AAVE dialect?

2. How do in-service teachers, support personnel, and/or administrators evaluate texts written in AAVE versus MAE as related to special education referral? 


\section{CHAPTER III: METHODS}

To embrace bidialectalism for AAVE and MAE speakers, a celebratory attitude towards African American culture is needed to validate the AAVE dialect (Lobeck, 2019) as a means to actively combat the negative feelings already associated with it by educators (Gupta, 2010; Newkirk-Turner et al., 2013). For this reason, a raciolinguistic perspective is the most ideal framework to consider for instruction that incorporates AAVE (Alim, Rickford, \& Ball, 2016) as it moves past the sociocultural theory (Vygotsky, 1978), past the sociolinguistic theory (Labov, 2001), and embraces an open acknowledgement of the intersection between race and language (Alim et al., 2016).

\section{Theoretical Framework}

With schools becoming progressively more diverse, it is imperative that educators employ evidence-based practices for effective instructional and assessment practices in relation to the culture and context of the student (Fallon et al., 2012). Within the field of disproportionality in special education, questions of whether students from dissimilar ethnic groups receive the interventions and supports that are needed across all types of settings persist. What is needed in the field of education in the analysis of disproportionality is a methodical approach of assessing disability that places social, cultural, and historical contexts at the center of the conversation. An approach such as this one will address questions about impact based on individual perspective, voice, and consequence (Ford et al., 2015; Skiba, Artiles, et al., 2015).

The prominent education scholar, William Tate, proposed the Critical Race Theory as a means to challenge school norms in the United States public school system and expose the racist underpinnings of standardized testing. Critical Race Theory has its origins in a legal response to the Civil Rights Movement and ways to address racial injustice seen through societal 
institutional and structural racism (Tate, 1996). Employing a sociocultural (Vygotsky, 1978), sociolinguistic (Labov, 2001), and raciolinguistic (Alim, Rickford, \& Ball, 2016) framework, along with Critical Race Theory (CRT) (Tate, 1996), researchers developed pedagogies at crosspurposes to dominant school norms designed to address the needs of CLD learners (Lynn et al., 2002). Given that, studying AAVE within these contexts seated in racism not only affirms discussions surrounding the AAVE dialect (Rickford et al., 2012) but also includes AAVE in discussions about instruction contrary to United States White public-school norms (Tate, 1996).

\section{Raciolinguistics}

The raciolinguistic theoretical framework openly identifies the connection between race and language (Alim et al., 2016) and may prove beneficial when having discussions about the historic marginalization of AAVE as a dialect (McKenna, 2013). Further, a raciolinguistic perspective may be instrumental when considering AAVE within the inseparable context of the longstanding maltreatment of African Americans who are the primary speakers of the dialect (Meyer, 2009). Moreover, raciolinguistics acknowledges that young AAVE speakers come to school with their own sociocultural and linguistically communicative behaviors (Seltzer \& Rios, 2018) that are historically viewed as negative (Gupta, 2010). Moreover, raciolinguistics considers the longstanding systemic oppression and discrimination of African Americans throughout United States history, beginning with slavery (Meyer, 2009). In response, it embraces a philosophy that works to promote AAVE speaking students' bidialectalism by way of codeswitching between AAVE and MAE in the classroom setting (Creese et al., 2010) while boosting self-pride and self-respect of African American culture (Campbell et al.,2017).

A goal of bidialectalsim for AAVE speakers by means of codeswitching not only calls for a celebratory attitude towards African American culture, it also calls for validation of the AAVE 
dialect (Lobeck, 2019) as a means to actively combat the negative feelings already associated with it by educators (Gupta, 2010; Newkirk-Turner et al., 2013). For this reason, a raciolinguistic perspective is the most ideal framework to consider for instruction that incorporates AAVE (Alim et al., 2016). A raciolinguistic philosophy moves past the sociocultural theory (Vygotsky, 1978), past the sociolinguistic theory (Labov, 2001), and embraces an open acknowledgement of the intersection between race and language (Alim et al., 2016). The raciolinguistic theory openly identifies the connection between race and language and readily recognizes that cultural linguistic ethnicity is both seen and heard (Alim et al., 2016). This framework may prove invaluable when having discussions about the historic marginalization of AAVE as a dialect (McKenna, 2013).

For this study, I built on traditions of research that engage in culturally responsive pedagogical analysis (Gay, 2002, 2010; Harmon, 2012; Ladson-Billings, 1995, 2000, 2006) using a raciolinguistic framework to analyze prejudicial notions based on language and race (Alim et al., 2016; Flores \& Rosa, 2015), reasoning that in-service educators are informed by the very discourse of White mainstream norms that are postulated within the United States public school system (Seltzer \& Rios, 2018). Raciolinguistics highlights the intersection of race and language and an understanding of how these indicators interact with institutions in education to give way to a naturalized perceived power of "normalized" Whiteness as an authoritative supremacy (Alim et al., 2016; Flores \& Rosa, 2015).

Further, when engaging in raciolinguistics as a theoretical framework for this study, I considered the ways that Whiteness and MAE are powerfully intertwined and mutually integrated and are "normalized" and embedded in the United States public school system (Seltzer \& Rios, 2018). This concept further shapes and engages in the complex possibilities of 
educators' subjective evaluation of students, particularly culturally linguistically diverse students who are African American and communicate using AAVE. Moreover, this also explores the potential consequences of those subjectivities and considers that these influences may in fact be a direct contribution to disproportionality in special education and the over-identification of African Americans in special education for disabilities that have subjective descriptions. The raciolinguistic framework in this study represents the complex concepts and processes that White "norms" regarding language.

\section{Research Design}

\section{Research Questions}

The research questions for this study were:

1. What are the perceptions of in-service teachers, support personnel, and/or administrators towards the AAVE dialect?

2. How do in-service teachers, support personnel, and/or administrators evaluate texts written in AAVE versus MAE as related to special education referral?

\section{Mixed Method Concurrent Design}

This study used a mixed method concurrent design (Creswell et al., 2017). Mixed method designs originated around the late 1980s and early 1990s based on the work of individuals in diverse fields such as evaluation, education, and sociology. In general, mixed method research designs involve the collection of both qualitative and quantitative data in response to research questions. For mixed method designs, both open-ended qualitative data and closed-ended quantitative data are collected and analyzed. Procedures for both qualitative and quantitative data collection are rigorously gathered through adequate sampling and various sources of information. 
A mixed method concurrent research design is often used in the field of education specifically to better understand long-term program goals. In a mixed method concurrent design, equal weight and emphasis is placed on both qualitative and quantitative data. The qualitative and quantitative data forms are integrated into the mixed method concurrent design analysis by merging, connecting, or embedding the data. For the mixed method concurrent research design, data is collected using a concurrent timing method to gather both quantitative and qualitative data simultaneously at the same time during a single phase of the study.

I chose the mixed method concurrent design because of its strength of drawing on both qualitative and quantitative research data without minimizing the limits of either datum set. This design provides a complex approach that appeals to novel research procedures. Also, a mixed method concurrent design is an ideal approach when the researcher has access to both quantitative and qualitative data designed to gain a more complete understanding of research questions that include (a) comparing different perspectives drawn from both quantitative and qualitative data, (b) explaining quantitative results with a qualitative follow-up data collection and analysis, and (c) developing a more complete understanding of changes that may need to occur for marginalized people groups through the combining of qualitative and quantitative data (Creswell et al., 2017).

This approach was beneficial for this study as it allowed for novel qualitative exploration and analysis how beliefs and opinions about AAVE explained quantitative analysis of educator participants' analyses of 3rd grade texts written in AAVE versus MAE through answering a yes/no questions. Further, the mixed method concurrent approach allowed for quantitative exploration regarding whether the participant would recommend student texts written in AAVE versus MAE for a special education evaluation while qualitatively analyzing why educators 
made those decisions at the same time. This allowed for a comparison of different perspectives drawn from both quantitative and qualitative data (Creswell et al., 2017).

Further, I investigated how participants' thoughts and ideas about AAVE informed their decision-making process when making referrals to special education. This was evident when I asked the participants an open-ended question to explain why they chose to refer or not to refer the student to special education. This qualitative follow-up data collection and analysis was useful as it was gathered to further explain quantitative results in the study of referrals decisions for special education evaluation. Exploring educators' responses to MAE and AAVE written vignettes (Appendix E) allowed me to analyze how educators explained their experiences with MAE- and AAVE-speaking students in the classroom setting provided a quantitative data set in the survey. This was particularly beneficial in analyzing and gaining an in-depth understanding of processes and practices educators might use when making decisions about special education referrals. Moreover, this data proved useful in developing a more complete understanding of implications choices made that may impact AAVE speaking students through the combined analysis of qualitative and quantitative data (Creswell et al., 2017).

The mixed method concurrent design was used to analyze the qualitative data of educator participants' attitudes and perceptions towards AAVE in the classroom and quantitative data of how educators evaluate texts written in AAVE and MAE as well as qualitative data related to the process educators use to make decisions about placing referrals to special education. This information was gathered via survey which participants completed simultaneously at the same time (Creswell et al., 2017). Specifically, to inquire about educator participants' perceptions and attitudes about AAVE, a survey using a 6-point Likert scale was used. The survey examined educators' general attitudes toward AAVE and educators' perceptions of AAVE as a legitimate 
dialect for use in an academic setting (Champion et al., 2012). The survey allowed for insight to developing a more complete understanding of how educators made sense of their experiences with AAVE speaking students both in general and in the classroom setting. In turn, this allowed for a deeper understanding of educators' thought processes related to practices with AAVE speaking students in the classroom.

\section{Selection of Participants}

I used voluntary response sampling and convenience sampling of educators to select participants for this study. Voluntary response sampling refers to a sample of participants who have voluntarily chosen to participate in a study, oftentimes because they have a strong opinion on the subject matter (Patton, 2015) . People volunteered themselves to participate in this study by responding to a public online survey on social media. In all, I invited educators to volunteer themselves to participate in the study for a total of 30 days, providing a mass call three times inviting educators to participate in this study online through social media.

Convenience sampling was also used to invite participants for this study. Convenience sampling refers to taking a sample from those individuals who are available (Patton, 2015). To do this, I distributed mass emails to educators who were publicly available through websites and lists associated with schools and organizations. In all, I emailed a total of 1,166 accounts made public through school and organization websites to invite educators to participate in this study and complete the survey. I prompted all 1,166 educators three times over a 30 day period to complete the study. Participants were required to live in the United States of America, provide informed consent, and be 18 years of age or older. Anyone not meeting these descriptors were excluded from the study. Participants were not required to be educators at the $3^{\text {rd }}$ grade level or the elementary setting to participate in this study. 


\section{Recruitment and Ethical Considerations}

Recruitment for participation occurred via social media and mass emailing (Appendix A). I was affiliated with some entities where recruitment occurred, including in-service educator(s) at school district(s) and practicing service(s). Demographics were obtained from participants, but no identifying information (e.g., name, identification number, etc.). Information that is publicly available, such as directory information, was also used for recruitment. Although no identifying information was gathered for this study because all participants were anonymous, all data were stored on my password-protected laptop. All data analysis occurred in a university or home office to maintain privacy and confidentiality.

Personnel working in education (i.e., administrators, professors, teachers, support personnel) had the choice as to whether their data could be included in the proposed project and the voluntary nature of the study was emphasized when the potential participants were recruited. Descriptive features (e.g., occupation, etc.) were used in the reporting of findings. All data were gathered anonymously to protect the identity of the participants. Any reports of findings only referred to participants by demographic descriptions that do not re-identify a participant (e.g., special education teacher). Due to the mass call for participation, participants varied in age, race, education, and history working within education.

All participants in the study were informed that participation was voluntary, and that they were free to withdraw from the study at any time. The survey was done in Qualtrics (Qualtrics; Version XM, 2020) using the anonymize function so that participant information including IP address could not be ascertained. There was a very slight chance that someone might be identifiable through a combination of job description (administrator, educator, etc.), and demographics (race, type of school, etc.). However, this chance was highly unlikely due to the 
anonymous nature of the survey, and the fact that I did not ask participants to identify their employer (e.g., schools, school districts, etc.). Responses were recorded and analyzed to identify themes and patterns.

Informed Consent. Informed consent (Appendix B) was addressed at the end of this study as there was some potential for deception in presentation of the written vignettes. This is because participants were not notified prior to reading the vignettes that the research study was centered around AAVE or that there was potential the vignettes were written in AAVE.

Participants were initially told that the study was about disproportionality in special education. However, participants were informed at the end of the study, during the debriefing, that the research was in fact regarding AAVE and disproportionality in special education and then they were asked if they consented to have their data included in the study. Participants were told about the true nature of the study during the debriefing at the end as opposed to the beginning to not potentially skew responses to results based on biased or influenced responses. I received a total of 60 responses to the online survey; however, given that the consent form for this study was at the end of the survey, only 41 participants completed the final debriefing and provided informed consent for their data to be included in this study.

\section{Participant Demographics}

A total of 41 participants completed the demographic questions and online survey and provided informed consent for this study. Participants in the study were in-service teachers, administrators, and support personnel. All participants were 18 years older serving in a U.S. public school setting. The majority of the participants were female (73\%), White or Caucasian (67\%), and had a Master's degree (68\%). Roles in PreK-12 Education included administrators (15\%), support personnel (20\%), and teachers (34\%). About a third of participants were 
classified as other $(32 \%)$, or 13 of the 41 total participants. I defined the category other as a variety of different professional roles, including adjunct faculty $(2 \%)$; behavior action/socialemotional learning team member $(2 \%)$; behavior consultant $(2 \%)$; board-certified behavior analyst (2\%); college classroom instructor (2\%); English language learner newcomers instructor $(2 \%)$; instructor for 4th-8th grade instrumental music $(2 \%)$; instructor for full inclusion district (2\%); math specialist (2\%); school-based specialist for special education (2\%); school counselors (5\%); and superintendent (2\%). Refer to Table 2 for complete information on the participant demographics.

\section{Table 2}

Educators' Demographic Profiles Response Frequencies and Percentages

\section{Item}

Gender

Female

Male

Racial/Ethnic Identification

Black or African American

White or Caucasian

Hispanic or Latino

Age Range

25-34 years

$35-44$ years

45-54 years

55-64 years

Highest Level of Education Completed

Bachelor's degree

Master's degree

Doctorate degree

\section{$\mathrm{N}(\%)$}

$30(73)$

$11(27)$

$11(26)$

$28(67)$

$3(7)$

9 (22)

$13(32)$

$15(37)$

4 (10)

4 (10)

$28(68)$

$9(22)$

Current Role in PreK-12 Education

Administrators

Director of Special Education

$6(15)$

$3(7)$

$3(7)$

$8(20)$

Support Personnel

$3(7)$

$1(2)$

$4(10)$

Teachers
Occupational Therapist

Speech-Language Pathologist 
General Education Classroom Teacher

Special education resource teacher

Other

Special education classroom teacher

\section{Grade Level currently working with in PreK-12}

Pre-Kindergarten

Kindergarten - 2nd Grade

3rd - 5th Grade

6th - 8th Grade

9th - 12th Grade
7 (17)

$4(10)$

$3(7)$

$13(32)$

$10(12)$

$25(29)$

$26(30)$

$18(21)$

$7(8)$

Risks. There were minimal foreseeable risks involved in participating in this study other than those encountered in day-to-day life. Potential risks included a breach of confidentiality, which is always a risk in data collection. There was also a potential social risk of employability or reputation should a breach of confidentiality occur. To reduce this risk, all data collected were anonymous. Additionally, there was a potential risk of coercion since I was recruiting participants within public schools where previously employed and/or currently affiliated. To minimize this risk of coercion, I was not a speech-language pathologist of record for any public schools in which the potential participants were employed at the time of recruitment.

The minimal benefits of participation included the satisfaction one might derive from contributing to the body of knowledge on service learning about AAVE. They were justified by the minimal risk of a breach of confidentiality. This was addressed by research personnel being adequately trained in how to maintain confidential participation and by using only demographic descriptors in the dissemination of findings.

Confidentiality. To minimize the risk of breach of confidentiality and ensure there is not a breach of confidentiality, I collected the data anonymously and I stored all linking codes to Qualtrics in a separate location from the data. The data was stored on a password-protected device and/or locked filing cabinet. I used all reasonable efforts to keep any provided personal 
information confidential so that re-identification did not occur. Information that may identify or potentially lead to re-identification were not released to individuals that were not on the research team. Demographic descriptors related to job description were used in dissemination (e.g., special education teacher, speech-language pathologist, etc.). However, when required by law or university policy, identifying information may be seen or copied by authorized individuals.

\section{Data Collection and Data Sources}

Data was collected using three tools (Appendices C, D, and E) which took approximately 20 minutes to complete. First, participants answered demographic questions to learn about their educational role in the district, years of experience, years in district, years of education, ethnicity, gender, etc. Participants had the option of Prefer Not to Respond whenever inclined. Second, participants read four typed writing samples of third graders written in: (a) AAVE and (b) MAE. The vignettes were presented in a randomized order for all participants. For each vignette, participants were asked, “Given this third-grader's writing sample, would you be inclined towards the special education referral process for this student?". Participants were asked to respond with: (a) yes or (b) no. After each response to a vignette, participants were asked the open-ended question, "Why did you choose to refer or not to refer this student to special education?"

During the initial email explaining the study, I invited participants and explained the research to recruit volunteers for the online study (Appendix A). After explaining the research via email using the attached recruitment script, participants were provided with the consent forms for electronic submission. Potential participants had the opportunity to complete the consent form at the time of the initial contact. Participation in this research is entirely voluntary and anonymous and participation will not impact employment or working relationships. I invited 
all who might be interested in participating in this study across the US who worked in education in the role of special education teacher, general education teacher, support personnel, and/or administrator.

Surveys, vignettes, and questionnaires completed for this study were analyzed immediately and will be destroyed five years after the research study by me. Likewise, after a period of five years, I will shred any hard copies of consent forms and data collection forms used in the system. All data associated with participants will be destroyed in accordance with university policy for standard educational practice.

\section{Instruments}

The purpose of this research study was to investigate whether educators would refer a student who communicated using AAVE in writing for a special education evaluation. For this study, in-service educators completed an online 25-item survey using a 6-point Likert scale regarding attitudes and perceptions of AAVE based on the work of Champion et al., (2012). Educators also read two vignettes written in AAVE and two vignettes written in MAE by $3^{\text {rd }}$ graders and made decision about referrals to special education based on the work of Common Core State Standards Initiative (2010), Fogel \& Ehri (2000), and Reading Rockets (2020). Lastly, participants were asked to explain why they made decisions to refer or not refer students for a special education after reading the vignettes.

Content-related survey, demographic questions, and written vignettes were reviewed by four professors in the education department at the university level as members I selected to participate on my dissertation committee. Upon agreement among the reviewers, the survey, questions, and vignettes were finalized and then piloted with a small sample of in-service educators representing the target population. 
Perception Survey. The survey used in this study was a modified version of the Language Attitude Scale (Ford, 1978) as modified by Champion et al. (2012). The perception survey was based on the work of Champion et al. (2012). The survey was designed to examine the educators' attitudes towards AAVE and their general perceptions of AAVE as a legitimate dialect for use in an academic setting. The survey was administered using an online survey platform, Qualtrics, to gain insight into the perceptions of education personnel of students who speak AAVE and its perceived impact on disproportionality in special education. The perception survey form is attached (Appendix C).

Champion et al. (2012) assessed the survey for internal reliability and found it to be highly reliable, with a Cronbach's alpha value of .93. Champion et al. (2012) judged internal and external validity of the survey as well. Construct, content, and face validity of the survey were deemed to be a fair representation of the general subject-matter the survey was designed to measure and representative of all aspects of the subject-matter the survey was designed to measure. Champion et al. (2012) considered criterion validity to be high based on results from the Language Scale Sample developed originally by Ford (1978).

The perception survey used in this study consisted of 25 questions using a 6-point Likert scale (Appendix C). Of the 25 questions on the survey, 15 questions examined the educators' general attitude toward AAVE while 10 question addressed the educators' perceptions of AAVE as a legitimate dialect for use in an academic setting (Champion et al., 2012). Four reviewers on my dissertation committee were asked to review the survey (Champion et al., 2012) to be sure that it was a fair representation of the general content the survey was designed to including the attitudes and perceptions educators hold of AAVE and AAVE use in the classroom setting. 
Demographic Questions. A demographic section of the survey gathered educators’ background including (a) gender, (b) racial/ethnicity identification, (c) age range, (d) highest level of education completed, (e) total years working in PreK-12, (f) number of professional development courses completed addressing reading-language arts, and (g) grade level currently working in PreK-12 (Appendix D). This information was collected to assess and identify possible patterns of responses to the survey and to find any commonalities and differences that might be presented across demographic background.

Vignettes. Data was also acquired through written vignettes based on the works of Common Core State Standards Initiative (2010), Fogel and Ehri (2000), Green (2002) and Reading Rockets, (2020). All four writings represented $3^{\text {rd }}$ grade students, but with differentiation in: (a) two vignettes written in AAVE and (b) two vignettes written in MAE. Participants were presented with two vignettes written in MAE and two vignettes written in AAVE to offer more than one opportunity to analyze literacy samples in each of the dialects and increase representation of each and increase opportunities for comparative results. Third grade was chosen for the written vignettes because third grade has been identified as the final year students are learning to read and instead are reading to learn. Further, researchers found that literacy skills at third grade had a direct correlation with long-term academic success (Hernandez et al., 2011; Lesnick et al., 2021).

None of the vignettes presented with any pathologies such as learning disabilities in writing. Organic vignettes were not used in this study from actual $3^{\text {rd }}$ graders; instead, I selected four vignettes from the literature based on perceived content characteristics. Characteristics that went into the decision of which vignettes to choose included the length of the sample. It was important that the vignette length was half page or less to guard against participant fatigue. Also, 
only vignettes that told a cohesiveness story where chosen, including a beginning, middle, and end. Further, no vignettes were presented with any grammatical or spelling errors as representative of typical features of $3^{\text {rd }}$ graders for MAE and AAVE found at Common Core State Standards Initiative (2010) and Green (2002). No errors were presented in the vignettes so that there were no ambiguous indicators of a disorder or pathology in any of the student writing samples.

Written vignettes were reviewed to ensure appropriate length and that qualities were consistent with a $3^{\text {rd }}$ graders' writing skills of typical development. Modifications were made to the written vignettes after there was disagreement within the committee about the length of the narratives, whether or not to include representations of a learning disability, and the option to include written scenarios surrounding the vignettes. When disagreements occurred, the committee discussed viable options and arrived at a consensus before proceeding. In the case of the length of written vignettes it was agreed that they should all only be a half page or less in length. The committee decided against including representation of a learning disability to allow for clearer comparisons between MAE and AAVE. Ideas regarding written scenarios were ultimately rejected due to the length of the survey and the concerns about participant fatigue.

\section{Data Analysis}

All responses from the 41 participants who gave consent to the survey were gathered and downloaded into a Microsoft Word and/or Excel spreadsheet for qualitative and/or quantitative analysis (Excel \& Word; Version 2018). All 41 responses to the 25-question, 6-point Likert scale survey were downloaded into Excel for quantitative analysis. Responses to why a special education referral was or was not made in response to the four written vignettes were 
downloaded into Word and then uploaded to the database Max Weber Qualitative Data Analysis (MAXQDA) for qualitative analysis (MAXQDA; Version 2020).

For the quantitative analysis, I used descriptive statistics to analyze overall responses to the questions about perceptions of AAVE, and responses based on specific demographic subgroups. Frequencies and percentages of responses were analyzed to examine overall trends, and whether there were any noticeable differences by subgroups. Due to a small participant pool, I did not conduct analyses for statistical significance.

For the qualitative analysis, I used MAXQDA to analyze text pertaining to educators' response to why a referral for special education was or was not made. I created Word documents using data downloaded from Qualtrics that displayed all of the text vignette responses for each of the nine professions and each of the four vignettes for a total of 13 documents analyzed. For every document, a set of variables were managed within the MAXQDA program for statistical analysis. After reviewing all the responses, I assigned codes to the responses, then placed each code into categories, and categories were further placed into themes and put into a codebook to allow for assessment in a concise way before confirmation, description, and definition of the codes.

\section{Research Questions}

\section{Research Question One: What are the perceptions of in-service teachers, support personnel,} and/or administrators towards the AAVE dialect?

Data gathered from this study related to demographic items were analyzed using descriptive statistical analysis to highlight frequency and percentage. The database Qualtrics provided descriptive analysis of demographic data that included age, gender, professional role, and all other demographic information collected in the survey. Qualtrics also provided 
descriptive analysis of responses on the Likert-scale survey, organized by question number and demographic information, and also organized responses for yes/no responses to the vignettes by name of the of the vignette and demographic information where frequency and percentage were provided for both. For the Likert-scale survey responses, Qualtrics provided calculations for each respondent for maximum, mean, standard deviation, variance, and numeric count for each response to the 25 questions.

Responses for each of these items were transferred into an excel spreadsheet for further analysis. I then manually organized the professional roles by categories of administrator, teacher, support personnel, and other. Other was provided as a categorical option to participants on the survey as a designation for profession with a designated space to specify profession by name.

I used excel functions AVERAGE and STDEV to calculate the mean and standard deviation, respectively, of professional categorical responses from teachers, administrators, support personnel, and other to the Likert scale survey questions. Number counts of yes/no responses to the vignettes by teachers, administrators, support personnel, and other were calculated manually by adding total number of responses by each professional discipline and separating them by category of teacher, administrator, and support personnel for vignettes written in AAVE versus MAE. Response frequencies of yes/no responses to the vignettes made by teachers, administrators, support personnel, and other were calculated manually by dividing total number of categorical professional responses by total number of responses for vignettes written in AAVE versus MAE. These analyses allowed me to answer the research question regarding the perceptions educators have towards the AAVE dialect. 


\section{Research Question Two. How do in-service teachers, support personnel, and/or}

administrators evaluate texts written in $A A V E$ versus $M A E$ as related to special education referral?

Response to vignettes were analyzed for common reasons educators provided for making the decision to refer or not to refer to special education, responses were coded, categorized, and themed to identify patterns (Miles et al., 2018). Participants' responses were already listed categorically by name of the vignette along with the title of professional role within Qualtrics. I then downloaded responses to a Microsoft Word documents containing responses from each of the four different vignettes and responses from each of the nine represented professional disciplines.

In the Qualtrics database, all data responses were organized according to specific role in education, gender, age, and all other demographic information gathered for this study. I spent a significant amount of time reading and re-reading responses, pondering the data to construct a thoughtful and organized narrative that provided a unified snapshot presented by the participants regarding reasons for considering a special education referral. This was done manually by hand to capture a true analysis that I could study and understand the responses of the participants.

During manual review, I worked to profile units of general meaning of the responses and units of meaning of the responses as it related to the research questions. This was done as an initial measure through preliminary review of results. This process allowed me to arrive at a basic level of understanding of the phenomenon of educators' decision-making process when referring students to special education (Hycner, 1985).

I then downloaded the text into 13 Microsoft Word documents, one for each participant's categorical professional role (9) and one for each written vignette (4). The 13 documents were 
then uploaded to the database MAXQDA for qualitative analysis. The MAXQDA database supports the mixed methods concurrent approach through analysis of text information within documents to manage system variable values. In MAXQDA, each document is a unit to be analyzed. The MAXQDA lexical search function, MAXDictio, enables word frequency searches of several documents at a time to conduct a quantitative text analysis. These results are placed into a table within MAXQDA which contains columns that can be used to compare word frequencies within and across documents. Then, a code analysis is completed through MAXQDA where codes are manually assigned with a word or words to anything considered worth marking, such as text or video segments (MAXQDA; Version 2020).

Codes can also be visualized by assigning individual colors to codes within and across documents. Visualization tools within MAXQDA allow for data connections in a comprehensible way, including use of the visual tool Code Matrix Browser, which displays which codes have been assigned to which documents. The Code Matrix Browser provides an overview of how many segments of each document have been assigned a specific code, for each existing code. Within the Code Matrix Browser, documents are listed in the columns while codes are listed in the rows, with display nodes viewed as squares, circles, or values. The Code Matrix Browser illustrates and analyzes the distribution of coded segments across documents (MAXQDA; Version 2020).

The 13 documents were then analyzed in MAXQDA using the lexical search function to find lexical repetitions to analyze for word frequencies within the documents. I reviewed the frequency of words and designated them as a significantly positive if the word occurred at least one time. Various common words that did not carry meaning such as prepositions, articles, and conjunctions were excluded from the frequency analysis as these occurred frequently through 
texts without true connotation of added perception. These variables were subsequently coded to total a preliminary number of 63 codes. A manual hand search revealed an additional 22 words that occurred and were added to the codebook to total 89 codes. I completed a manual review of all 89 codes and identified repetitions, redundancies, and similar derivations within codes and further combined codes. In the end, a total of 27 codes were identified and entered into the codebook.

Word responses of color-coded narrations were displayed using the computer program MAXQDA which highlighted various codes. After identifying the 27 codes and highlighting them on the 13 word doc embedded within the MAXQDA system, I manually grouped specific codes into recurring categories which answered the presenting research question for this study regarding how in-service educators evaluated texts written in AAVE versus MAE as related to special education referral. The 27 codes were manually grouped into a total of 11 categories based on topic similarities to further organize and systematize the data and further group analytical codes into themes. The 11 categories identified included: variable considerations, language system, mode of communication, discourse, work impressions, student measures, ability, educational support, cultural linguistics, educational services, and remediation.

The 11 categories were manually grouped into a total of four themes based on existing themes I recently reviewed in the literature (Skiba et al., 2013). Four themes were identified in this study, all of which were consistent with four themes previously identified by Skiba, Simmons et al. (2006). Therefore, I named the four themes in the same manner as Skiba, Simmons et al. (2006): (a) additional information needed, (b) general education factors, (c) perspectives on diversity, and (d) special education process. Themes were color-coded within MAXQDA according to their grouped 11 categories Skiba, Simmons et al. (2006). It is 
important to note that I was mindful and aware of the themes derived by Skiba, Simmons et al. (2006) while I was analyzing the data for this study.

Within MAXQDA, themes, categories, codes, and definitions were then developed into a codebook to represent all responses to vignettes made by educators whether a recommendation for special education was made or not. Codes are primarily used to identify and categorize similar data so that the research can find and cluster themes into segments related to a particular research question. The display of condensed chunks is an initial step when analyzing the data and drawing conclusions. (Miles et al., 2018).

I identified a total of 27 codes, 11 categories and four themes. It is important to note that themes, categories, and codes were not developed or analyzed as positive or negative in relation to perception or attitudes towards AAVE, but merely as existence of a determining factor during the educators' decision-making process when making a referral for special education. Also, at times, sentence responses were representative of more than one code, category, or theme and applied accordingly.

Themes were then compared and contrasted for common patterns using the MAXQDA feature analysis summary grid to complete a systematic analysis of all responses across professional roles and written vignettes. To complete this compare and contrast, I focused on what the participants said verbatim in quotes as was done throughout this analysis to develop codes for the responses. This systematic analysis of all coded responses was completed to develop a concise summary of responses based on written vignettes of AAVE versus MAE; professional category of administrators, teachers, and support personnel; and specific professional role of speech-language pathologist, general education classroom teacher, etc. This allowed me to present this qualitative data of themes, categories, and codes in a relatable and 
understandable way to answer the research question of how educators evaluate texts written in AAVE versus MAE as related to special education referral. For a complete list of themes, categories, codes, and definitions, related to responses of vignettes written in both MAE and AAVE, see Table 3.

Table 3

Codebook Framework and Definitions

\begin{tabular}{|c|c|c|c|}
\hline Theme & Category & Code & Definition \\
\hline Additional & Variable & Factors & Named factors to consider \\
\hline Information & \multirow[t]{4}{*}{ Considerations } & Reasons & Possible explanation provided \\
\hline \multirow[t]{3}{*}{ Needed } & & Not Enough & Not enough information provided \\
\hline & & Information & \\
\hline & & Time & Questioned how long struggling \\
\hline General & \multirow[t]{5}{*}{ Language System } & Orthography & Spelling \\
\hline Education & & Grammar & Grammar \\
\hline \multirow[t]{15}{*}{ Factors } & & Verbs & Verb tense \\
\hline & & Syntax & Sentence structure \\
\hline & & Semantics & English conventions \\
\hline & \multirow{3}{*}{$\begin{array}{l}\text { Mode of } \\
\text { Communication }\end{array}$} & Alphabetic & Writing \\
\hline & & & \\
\hline & & Linguistic & Speaking \\
\hline & \multirow[t]{2}{*}{ Discourse } & Story & Narrative structure \\
\hline & & Topic & Story topic \\
\hline & \multirow[t]{2}{*}{ Work Impressions } & Positive & Accuracy and normalcy \\
\hline & & Negative & Inaccuracy or concern \\
\hline & \multirow[t]{2}{*}{ Student Measures } & Grade & Grade level \\
\hline & & Age & Age level \\
\hline & \multirow[t]{2}{*}{ Ability } & Disability & Presence of disability \\
\hline & & Giftedness & Presence of giftedness \\
\hline & $\begin{array}{l}\text { Educational } \\
\text { Support }\end{array}$ & System of Support & RtI and/or MTSS \\
\hline \multirow{3}{*}{$\begin{array}{l}\text { Perspectives } \\
\text { on Diversity }\end{array}$} & \multirow{3}{*}{$\begin{array}{l}\text { Cultural } \\
\text { Linguistics }\end{array}$} & Diversity & Diverse Americans and ELL \\
\hline & & Dialect & Dialectal difference \\
\hline & & AAVE & AAVE, AAE, or BE \\
\hline Special & Educational & Special Education & Qualification for special education \\
\hline Education & Services & & \\
\hline Process & Remediation & Proposal & Referral for evaluation \\
\hline
\end{tabular}


Treatment Interventions and lessons

Assistance Help and support

Note. $A A V E=$ African American Vernacular English; $A A E=$ African American English; $B V E=$ Black Vernacular English; ELL = English Language Learner; RtI = Response to Intervention; MTSS $=$ Multi-Tiered System of Supports

Additional Information Needed. The theme additional information presented with the least number of categories with only one, called variable considerations. There were four codes identified with that category including: factors, reasons, not enough information, and time. The code factors referred to any named factor that was proposed for consideration. The code reasons referred to specific reasons that were provided to explain why or why not a referral was made. The code not enough information designated that the writer stated there was not enough information provided to make a decision. The code time referred to questions or statements that mentioned how long the student had been struggling with difficulties as a determining factor of whether or not a referral was warranted. These references were not analyzed as positive or negative, but merely as existence of a determining factor during the decision-making process when making a referral to special education.

General Education Factors. The theme of general education factors presented with the largest number of categories which included a total of six categories, all of which had more than one code. The six categories included in general education factors included language system, mode of communication, discourse, work impressions, students' measures, ability, and educational support. These categories were included as general education factors because they are common determining factors when educators are assessing academic performance (Met, 2008). The category language system included the codes orthography, grammar, verbs, syntax, and semantics. The code orthography was defined as spelling, the code grammar specified 
grammatical structure, the code verbs specified verb tense, the code syntax referred to sentence structure, and the code semantics denoted English language conventions. The category mode of communication included the code alphabetic defined as writing and the code linguistic defined as speaking. The category discourse included the code story to designate narrative structure and the code topic referred to the topic of the story. The category work impressions included the code positive to refer to statements about accuracy or normalcy and the code negative to refer to inaccuracy or concern. The category student measures included the code grade to refer to grade level and age to refer to age level. The category ability included the code disability to refer to mention of the presence of a disability and the code giftedness to refer to mention of presence of giftedness. The category education support referred to the one code of system of support which included Response to Intervention (RtI) and Multitiered System of Support (MTSS).

Perspectives on Diversity. The theme perspectives on diversity presented with only one category of cultural linguistics. That category was broken down into three categories: diversity, dialect, and AAVE. The code diversity referred to any mention of diverse Americans, including English Language Learners (ELL). The code dialect referred to any mention of dialectal difference and the code AAVE referred to any reference to AAVE or any other names used to refer to the same dialect, including African American English (AAE), Black Vernacular English (BVE), or Ebonics. References were not analyzed as positive or negative, but merely as existence of a determining factor during the decision-making process.

Special Education Process. The theme of special education process encompassed categories related to educational services or remediation. These two categories were further broken down into the codes: special education, proposal, treatment, and assistance. The code special education was used to refer to written responses that pertained to references to 
qualification of special education. The code proposal was used to reference responses related to referral for an evaluation. The code treatment referred to the need for interventions and lessons. The code assistance referred to the participant reference to the need for help and support. As stated, references were not analyzed as positive or negative, but merely as existence of a determining factor during the decision-making process for referral to special education

\section{Summary}

The theoretical framework for this study was raciolinguistics (Alim et al., 2016) to analyze ways that Whiteness and MAE are intertwined and embedded in the United States public school system (Seltzer \& Rios, 2018). This concept shapes and engages in the complex possibilities of educators' subjective evaluation of African American students who communicate using AAVE, which may in turn be directly contributing to the over-identification of African Americans as having a disorder and referring them to special education. A mixed method concurrent design was used to analyze educator participants' attitudes and perceptions towards AAVE in the classroom, how educators evaluate texts written in AAVE and MAE, and the process educators used to make decisions about placing referrals to special education (Merriam et al., 2016). Data was collected via Qualtrics.com using a 25-question survey, demographic questions, and written vignettes in MAE and AAVE. There were 41 participants in the study that included in-service administrators, teachers, and support personnel. Data was analyzed quantitatively using the online database Qualtrics and excel spreadsheets to identify frequencies, percentages, means, and standard deviation of responses. Data was analyzed qualitatively using the computer program database MAXQDA to identify codes, categories, and themes. 


\section{CHAPTER IV: FINDINGS}

\section{Purpose of the Study}

The purpose of this study was to investigate the special education referral process and gain a better understanding of the causes of disproportionality in special education for AAVE speakers who communicate using AAVE in writing. Specifically, I hoped to gain insight into educators' perceptions of AAVE and its use in the classroom setting. In this section, I present the findings to the research questions posed in the study. I begin by restating the research questions, followed by a description of the survey, vignettes, quantitative measures, and qualitative measures.

\section{Research Questions}

The research questions for this study were:

1. What are the perceptions of in-service teachers, support personnel, and/or administrators towards the AAVE dialect?

2. How do in-service teachers, support personnel, and/or administrators evaluate texts written in AAVE versus MAE as related to special education referral?

\section{Perception Survey}

Of the 25-question survey, question numbers $1,2,3,4,6,7,8,11,12,13,17,18,21,24$, and 25 examined the educators' general attitude toward AAVE. Question numbers 5, 9, 10, 14, $15,16,19,20,22$, and 23 addressed the educators' perception of AAVE in the classroom setting. The survey used a 6-point Likert scale where 1 represented "strongly disagree", 2 represented “disagree", 3 represented "slightly disagree", 4 represented "slightly agree”, 5 represented "agree" and 6 represented "strongly agree" (Champion et al., 2012). 
Of the 41 responses provided for the perception survey, results showed that educators' perceptions of the dialect were positive overall. Percentages and frequencies of responses are depicted in Table 4. The survey data were first analyzed to assess in-service educators' attitudes toward AAVE then to address the educators' perceptions of AAVE as a legitimate dialect for use in an academic setting. This analysis was done using data listing frequencies and percentages of participants' responses on survey items depicted in Table 4. Overall, results were mixed with both negative and positive views of educators' general attitude and perspectives towards AAVE reported along with educators' perceptions of AAVE as a legitimate dialect for use in an academic setting. Then, these two clusters of data from Table 4 were further analyzed by professional category through calculated means for each survey items. Below is a description of the results from the survey given these two descriptive clusters from the survey along with overall averages by professional role.

Of the 41 respondents to the survey, $81 \%$, or 33 respondents, perceived that "AAVE is a clear, thoughtful, and expressive language". Also, 83\%, or 34 respondents, did not perceive that "AAVE is a misuse of MAE" and the same number of respondents perceived that "AAVE is as effective for communication as is MAE". Also, $93 \%$, or 38 respondents, disagreed with the statement that "AAVE is an inferior language system". However, 39\%, or 16 respondents, perceived "AAVE as having a faulty grammar system" and $30 \%$, or 12 respondents, perceived that "AAVE sounds sloppy". Further, of those same 41 respondents, only $37 \%$ or 15 respondents perceived that "AAVE sounds as good as MAE", which means that $63 \%$, or 26 respondents, did not perceive that "AAVE sounds as good as MAE". See Table 4 for additional information.

Further, 44\%, or 18 respondents indicated that "AAVE must be accepted if pride is to develop among African Americans" and 53\%, or 22 respondents, did not perceive that "AAVE 
must be accepted if pride is to develop among African Americans". One respondent (2\%) did not respond to this survey item. This is the only survey question item that garnered a "no response". Also, 46\%, or 19 respondents, did not perceive that "AAVE sounds cool”.

\section{Table 4. Educators' Attitudes toward AAVE: Response Percentages and Frequencies}

\begin{tabular}{lcccccc}
\hline Item & $\begin{array}{c}\text { Strongly } \\
\text { Disagree } \\
(\%)\end{array}$ & $\begin{array}{c}\text { Disagree } \\
(\%)\end{array}$ & $\begin{array}{c}\text { Slightly } \\
\text { Disagree } \\
(\%)\end{array}$ & $\begin{array}{c}\text { Slightly } \\
\text { Agree } \\
(\%)\end{array}$ & $\begin{array}{c}\text { Agree } \\
(\%)\end{array}$ & $\begin{array}{c}\text { Strongly } \\
\text { Agree } \\
(\%)\end{array}$ \\
\hline $\begin{array}{l}\text { 1. AAVE is a } \\
\text { misuse of }\end{array}$ & $17(42)$ & $10(24)$ & $7(17)$ & $3(7)$ & $2(5)$ & $2(5)$ \\
MAE. & & & & & & \\
$\begin{array}{l}\text { 2. AAVE is a } \\
\text { clear, } \\
\text { thoughtful, and } \\
\text { expressive } \\
\text { language. }\end{array}$ & $0(0)$ & $5(12)$ & $3(7)$ & $14(34)$ & $8(20)$ & $11(27)$ \\
$\begin{array}{l}\text { 3. AAVE has a } \\
\text { faulty grammar } \\
\text { system. }\end{array}$ & $10(24)$ & $8(20)$ & $7(17)$ & $11(27)$ & $4(10)$ & $1(2)$ \\
$\begin{array}{l}\text { 4. Continued } \\
\text { usage of }\end{array}$ & $16(39)$ & $11(27)$ & $11(27)$ & $0(0)$ & & \\
AAVE would \\
accomplish \\
nothing \\
worthwhile for \\
society.
\end{tabular}


7. AAVE is

$3(7)$

$1(2)$

$15(37)$

$12(29)$

$8(20)$

$2(5)$

cool.

8. AAVE is as

$2(5)$

$2(5)$

$3(7)$

$9(22)$

16 (39)

$9(22)$

effective for

communication

as is MAE.

9. If use of

$1(2)$

$2(5)$

4 (10)

$13(32)$

$14(34)$

7 (17)

AAVE were

encouraged, speakers of

AAVE would

be more

motivated to

achieve

academically.

10. In a

predominantly

$2(5)$

$1(2)$

9 (22)

$11(27)$

$11(27)$

7 (17)

African

American

school, AAVE

as well as

MAE should be

taught.

11. Widespread

$0(0)$

$2(5)$

4 (10)

$18(44)$

10 (24)

7 (17)

acceptance of

AAVE is

imperative.

12. AAVE

$18(44)$

$15(37)$

5 (12)

$0(0)$

$2(5)$

$1(2)$

should be

considered a

bad influence

on American

culture.

13. AAVE

$1(2)$

5 (12)

$16(39)$

$2(5)$

$10(24)$

$6(15)$

must be

accepted if

pride is to

develop among

African

Americans. 


\begin{tabular}{|c|c|c|c|c|c|c|}
\hline $\begin{array}{l}\text { 14. Attempts to } \\
\text { eliminate } \\
\text { AAVE in } \\
\text { schools results } \\
\text { in situations } \\
\text { that can be } \\
\text { psychologically } \\
\text { damaging to } \\
\text { African } \\
\text { American } \\
\text { children. }\end{array}$ & $3(7)$ & $1(2)$ & $4(10)$ & $5(12)$ & $20(49)$ & $8(20)$ \\
\hline $\begin{array}{l}15 . \text { When } \\
\text { teachers reject } \\
\text { the native } \\
\text { language of a } \\
\text { student, they } \\
\text { do him/her } \\
\text { great harm. }\end{array}$ & $0(0)$ & $2(5)$ & 7 (17) & $6(15)$ & $10(24)$ & $16(39)$ \\
\hline $\begin{array}{l}\text { 16. One of the } \\
\text { goals of the } \\
\text { American } \\
\text { school system } \\
\text { should be the } \\
\text { standardization } \\
\text { of the English } \\
\text { language. }\end{array}$ & $6(15)$ & $11(27)$ & $6(15)$ & $11(27)$ & $6(15)$ & $1(2)$ \\
\hline $\begin{array}{l}\text { 17. AAVE } \\
\text { should be } \\
\text { discouraged. }\end{array}$ & $9(22)$ & $11(27)$ & $15(37)$ & $4(10)$ & $2(5)$ & $0(0)$ \\
\hline $\begin{array}{l}\text { 18. AAVE } \\
\text { should be } \\
\text { accepted } \\
\text { socially. }\end{array}$ & $0(0)$ & $2(5)$ & $2(5)$ & $14(34)$ & 7 (17) & $16(39)$ \\
\hline $\begin{array}{l}\text { 19. Acceptance } \\
\text { of AAVE by } \\
\text { teachers will } \\
\text { lead to a } \\
\text { lowering of } \\
\text { standards in } \\
\text { school. }\end{array}$ & $13(32)$ & $14(34)$ & $4(10)$ & 7 (17) & $3(7)$ & $0(0)$ \\
\hline
\end{tabular}




\begin{tabular}{|c|c|c|c|c|c|c|}
\hline $\begin{array}{l}20 \text {. The } \\
\text { scholastic level } \\
\text { of a school will } \\
\text { fall if teachers } \\
\text { allow AAVE to } \\
\text { be spoken. }\end{array}$ & $13(32)$ & $18(44)$ & $6(15)$ & $3(7)$ & $1(2)$ & $0(0)$ \\
\hline $\begin{array}{l}\text { 21. AAVE is } \\
\text { an inferior } \\
\text { language } \\
\text { system. }\end{array}$ & $17(42)$ & $9(22)$ & $12(29)$ & $2(5)$ & $1(2)$ & $0(0)$ \\
\hline $\begin{array}{l}\text { 22. A teacher } \\
\text { should correct } \\
\text { a student's use } \\
\text { of AAVE. }\end{array}$ & $3(7)$ & 7 (17) & $11(27)$ & $16(39)$ & $4(10)$ & $0(0)$ \\
\hline $\begin{array}{l}\text { 23. One } \\
\text { successful } \\
\text { method for } \\
\text { improving the } \\
\text { learning } \\
\text { capacity of } \\
\text { speakers of } \\
\text { AAVE would } \\
\text { be to replace } \\
\text { their dialect } \\
\text { with MAE. }\end{array}$ & $9(22)$ & $13(32)$ & $10(24)$ & $6(15)$ & $3(7)$ & $0(0)$ \\
\hline $\begin{array}{l}\text { 24. AAVE } \\
\text { sounds sloppy. }\end{array}$ & $7(17)$ & $10(24)$ & $12(29)$ & $8(20)$ & $4(10)$ & $0(0)$ \\
\hline $\begin{array}{l}25 \text {. The sooner } \\
\text { we eliminate } \\
\text { AAVE the } \\
\text { better. }\end{array}$ & $25(61)$ & $4(10)$ & $8(20)$ & $3(7)$ & $1(2)$ & $0(0)$ \\
\hline
\end{tabular}

Note: A total of 41 educators participated in the study and were allowed to skip questions. Percentages appear in parentheses.

Below is a summary of the mean categorical results of the survey based on the 41 responses from in-service teachers, support personnel, and administrators who completed the survey. A score of three is indicative of an educator slightly disagreeing with a statement on the 
survey. Mean scores depicted in Table 5 designate averages of the scores derived by educators' responses to survey items. Because three represented "slightly disagree" in this survey, the mean score of 2.84 as shown for administrators in Table 5 represents that, on average, administrators disagreed with the presented statement of "AAVE is a misuse of MAE" but with a wide range of variance of 1.56. See Table 5 for complete mean scores and standard deviations for educators who completed the survey. Frequency, percentage, and mean scores reveal mixed results. 


\begin{tabular}{|c|c|c|c|c|}
\hline Item & Administrators & $\begin{array}{l}\text { Support } \\
\text { Personnel }\end{array}$ & Teachers & Other \\
\hline AAVE is a misuse of MAE & $2.84(1.56)$ & $1.67(0.27)$ & $2.46(1.04)$ & $2.11(1.2)$ \\
\hline AAVE is clear & $4.83(0.71)$ & $4.89(0.31)$ & $4.49(0.98)$ & $3.89(1.29)$ \\
\hline AAVE has faulty grammar & $2.84(1.04)$ & $2.78(0.42)$ & $3.02(1.27)$ & $3(1.15)$ \\
\hline AAVE accomplish nothing & $2(0.71)$ & $1.67(0.27)$ & $2.19(1.14)$ & $2.44(1.17)$ \\
\hline AAVE should be allowed in classrooms & $4.17(0.65)$ & $4.83(0.77)$ & $4.84(1.05)$ & $4(0.94)$ \\
\hline AAVE sounds as good as MAE & $3.67(0.65)$ & $3.78(0.94)$ & $3.62(1.41)$ & $2.78(1.03)$ \\
\hline AAVE is cool & $4(0.86)$ & $3.97(.68)$ & $3.50(1.02)$ & $3.67(0.94)$ \\
\hline AAVE is effective & $4.67(0.65)$ & $4.44(0.68)$ & $4.81(0.95)$ & $4.33(1.15)$ \\
\hline AAVE motivates academic achievement for African Americans & $4.34(.65)$ & $4.47(0.78)$ & $4.68(0.95)$ & $4.22(1.31)$ \\
\hline AAVE should be taught in school & $4(0.86)$ & $4.67(0.9)$ & $4.59(1.08)$ & $3.89(0.87)$ \\
\hline AAVE acceptance is imperative & $4(0.47)$ & $5.06(0.48)$ & $4.55(0.98)$ & $4.11(0.87)$ \\
\hline AAVE is a bad for African American culture & $2.5(1.49)$ & $1.64(0.3)$ & $1.8(0.85)$ & $2(0.82)$ \\
\hline AAVE acceptance will boost pride for African Americans & $4(1.1)$ & $4.39(0.92)$ & $4.07(1.23)$ & $3.44(1.17)$ \\
\hline Eliminating AAVE is damaging to African Americans & $4(1.5)$ & $5.19(0.3)$ & $5.15(0.67)$ & $3.78(1.4)$ \\
\hline Rejection of native language is harmful & $4.67(0.88)$ & $5.03(0.86)$ & $5.17(0.76)$ & $4.33(1.05)$ \\
\hline Schools should standardize the English language & $3.17(1.04)$ & $2.83(0.65)$ & $2.96(1.21)$ & $3.56(1.26)$ \\
\hline AAVE should be discouraged & $2.33(0.65)$ & $2.36(0.46)$ & $2.67(1.35)$ & $2.67(0.67)$ \\
\hline AAVE should be accepted socially & $4.5(0.71)$ & $5.58(0.42)$ & $4.91(0.96)$ & $4.22(1.13)$ \\
\hline AAVE acceptance will lower school standards & $2(0.86)$ & $1.83(0.44)$ & $2.07(0.98)$ & $3.11(1.2)$ \\
\hline AAVE spoken in schools will lower scholastic level & $1.84(0.65)$ & $1.72(0.45)$ & $1.8(0.79)$ & $2.44(0.68)$ \\
\hline AAVE is inferior & $1.84(0.88)$ & $1.92(0.71)$ & $2.09(1.04)$ & $2.11(0.74)$ \\
\hline AAVE should be corrected by teachers & $3.17(1.04)$ & $3.19(0.52)$ & $3.07(1.05)$ & $3.78(0.79)$ \\
\hline AAVE should be replaced with MAE by teachers & $2(1.1)$ & $2.56(0.56)$ & $2.6(0.97)$ & $2.22(0.92)$ \\
\hline AAVE sounds sloppy & $2.34(0.88)$ & $3.06(0.71)$ & $2.74(0.87)$ & $3(1.15)$ \\
\hline AAVE should be eliminated & $1.34(0.47)$ & $1.67(0.27)$ & $2.09(1.04)$ & $1.67(1.05)$ \\
\hline
\end{tabular}

Note: Mean scores are based on participants' responses using a Likert Scale from 1-6. 


\section{Administrators}

The six (15\%) administrators who responded to the survey yielded positive general attitudes towards AAVE overall. Administrators presented with mean scores of 2.84, 4.0, 4.0, and 2.34, respectively, to survey items related to AAVE having a faulty grammar, being cool, boosting pride for African Americans, and sounding sloppy. These mean scores indicate that administrators did not express attitudes towards AAVE as having faulty grammar or sounding sloppy. Make note that these results should be interpreted with caution since scale items were not reverse-coded. Further, mean scores indicated that administrators presented with attitudes that AAVE is not only cool or remarkable but that accepting AAVE as a legitimate dialect may in fact boost pride amongst African Americans. Overall, administrators presented with the most positive general attitudes towards AAVE in disagreeing that AAVE sounds sloppy, with a mean score of 2.38, as compared to support personnel and teachers with mean scores of 3.06 and 2.74, respectively. Also, administrators are the only professional who expressed that AAVE is cool on the survey, with a mean score of 4.0, while support personnel presented with a mean score of 3.97 and teachers presented with a mean score of 3.5.

\section{Support Personnel}

The eight (20\%) support personnel who responded to the survey yielded mixed general attitudes towards AAVE overall. Support personnel presented with mean scores of 2.78, 4.39, and 3.09, respectively, to survey items related to AAVE having a faulty grammar, boosting pride for African Americans, and sounding sloppy. These mean scores indicate that support personnel did not express attitudes towards AAVE as having faulty grammar or sounding sloppy. Also, mean scores indicated that support personnel presented with attitudes that AAVE is as a 
legitimate dialect and may boost pride amongst African Americans. Support personnel presented with the highest general attitudes toward AAVE as it relates to not being a misuse of MAE, with a mean score of 1.67 compared to 2.84 for administrators and 2.46 for teachers. However, support personnel presented with attitudes that AAVE is not cool or remarkable with a mean score of 3.97, compared to administrators' mean score of 4 and teachers' mean score of 3.5 .

\section{Teachers}

The $14(34 \%)$ teachers who responded to the survey yielded mixed general attitudes towards AAVE overall. Teachers presented with mean scores of 3.02, 4.07, 2.74, and 2.46 respectively, to survey items related to AAVE having a faulty grammar, boosting pride for African Americans, sounding sloppy, and AAVE being a misuse of MAE. These mean scores indicate that teachers did not express attitudes towards AAVE as having faulty grammar or sounding sloppy or being a misuse of MAE. Also, mean scores indicated that teachers presented with attitudes that AAVE is as a legitimate dialect and may boost pride amongst African Americans. Teachers presented with the highest general attitudes toward AAVE as it relates to having a faulty grammar, with a mean score of 3.02 compared to 2.84 for administrators and 2.78 for support personnel. Also, teachers presented with most negative attitudes that AAVE is not cool or remarkable with a mean score of 3.5 , compared to administrators' mean score of 4 and teachers' mean score of 3.97.

\section{Educators' Perceptions of AAVE as a Legitimate Dialect for Use in an Academic Setting}

Of the 41 respondents to the survey, $74 \%$, or 30 respondents, perceived "teachers should allow African American students to use AAVE in the classroom”. Of those same 41 respondents, $83 \%$, or 34 respondents perceived that "if use of AAVE were encouraged, speakers of AAVE would be more motivated to achieve academically". Also, 71\%, or 29 respondents, 
perceived that "in a predominantly African American school, AAVE as well as MAE should be taught" and $81 \%$, or 33 respondents agreed that "attempts to eliminate AAVE in schools results in situations that can be psychologically damaging to African American children.”. However, $49 \%$, or 20 respondents indicated that a teacher should correct a student's use of AAVE; although, only $22 \%$, or nine respondents agreed that "one successful method for improving the learning capacity of speakers of AAVE would be to replace their dialect with MAE.”.

Of the 41 responses, mean score responses by categorical professional role were further analyzed below. During further analysis of the survey data, particular attention was related to generalized responses that presented as described and exemplified in the preceding two paragraphs and in Table 4. In particular, data items were further analyzed to assess in-service educators' perceptions of AAVE as legitimate dialect for us in the classroom setting. This was completed using data scores reported on the Likert scale for each item by each participant and then organized by categorical professional role using an excel spreadsheet.

Mean scores were calculated to identify the average response of each item related to each professional role. Mean responses overall of perceptions of AAVE were revealed to be perceptions of AAVE as a legitimate dialect for use in the classroom setting yielding mean scores of 3.34, 3.63, and 3.68 from administrators, support personnel, and teachers, respectively. This indicates that the educators surveyed $(n=41)$ generally disagreed with much of the negative statements made about AAVE in the survey. The following paragraphs provide specific information regarding responses made by administrators, support personnel, and teachers. In general, administrators, support personnel, and teachers presented with consistent positive general perceptions of AAVE as legitimate and supported use in the classroom setting based on mean scores derived from the survey. 


\section{Administrators}

The six (15\%) administrators who responded to the survey yielded positive perceptions of AAVE as legitimate dialect for use in the classroom setting overall. Administrators presented with mean scores of $4.17,4.34$, and 4.0, respectively, to survey items related to AAVE being allowed in classrooms, motivating academic achievement for African Americans, and teaching AAVE in school. This means that administrators agreed that AAVE should be allowed in classrooms, that it may motivate academic achievement for African Americans, and that AAVE should be taught in schools. Also, mean scores of 4.0 and 2.0, respectively, indicated that administrators presented with attitudes that eliminating AAVE is damaging to African Americans and that acceptance of AAVE acceptance would not lower school standards. Further, administrators presented with the strongest response against AAVE being replaced with MAE by teachers with a standard score of 2.0 as compared to support personnel and teachers who had mean scores of 2.56 and 2.6 , respectively.

\section{Support Personnel}

The eight (20\%) support personnel who responded to the survey yielded positive perceptions of AAVE as legitimate dialect for use in the classroom setting overall. Support personnel presented with mean scores of $4.83,4.47$, and 4.67 , respectively, to survey items related to AAVE being allowed in classrooms, motivating academic achievement for African Americans, and teaching AAVE in school. This means that support personnel agreed that AAVE should be allowed in classrooms, that it may motivate academic achievement for African Americans, and that AAVE should be taught in schools. Also, mean scores of 5.19 and 2.56, respectively, indicated that support personnel presented with attitudes that eliminating AAVE is damaging to African Americans and AAVE should not be replaced with MAE by teachers. 
Further, support personnel presented with the strongest response in agreeance that acceptance of AAVE would not lower school standards with a mean score of 1.83 as compared to 2.0 and 2.07, respectively, for administrators and teachers. Also, support personnel presented with the strongest response that eliminating AAVE is damaging to African Americans with a mean score of 5.19, compared to 4.0 and 5.15 from administrators and teachers, respectively.

\section{Teachers}

The 14 (34\%) teachers who responded to the survey yielded perceptions of AAVE as legitimate dialect for use in the classroom setting overall. Teachers presented with mean scores of 4.84, 4.68, and 4.59, respectively, to survey items related to AAVE being allowed in classrooms, motivating academic achievement for African Americans, and teaching AAVE in school. This means that teachers agreed that AAVE should be allowed in classrooms, that it may motivate academic achievement for African Americans, and that AAVE should be taught in schools. Teachers rejected the notion that schools should standardize the English language with a mean score of 2.96 and teachers further agreed that the rejection of native language is harmful with a mean score of 5.17. Also, mean scores of 5.15 and 2.6, respectively, indicated that teachers presented with attitudes that eliminating AAVE is damaging to African Americans and AAVE should not be replaced with MAE by teachers. Further, teachers presented with the strongest response in agreement that AAVE should be allowed in classrooms with a mean score of 4.84 as compared to 4.17 and 4.83 , respectively, for administrators and support personnel. Also, teachers presented with the strongest response in agreeance that use of AAVE motivates academic achievement for African Americans with a mean score of 4.68 as compared to 4.34 and 4.47, respectively, for administrators and support personnel. 


\section{Educators' Evaluation of AAVE versus MAE Texts Related to Special Education Referral}

There was a total of $15(9 \%)$ referrals made for special education services for the total of 164 possible referrals for both the vignettes written in AAVE and MAE combined. There were a total of 82 possible referrals for vignettes written in AAVE, with 41 referrals possible for each of the two vignettes. Likewise, there were a total of 82 possible referrals for vignettes written in MAE, with 41 referrals possible for each of the two vignettes. A total of $13(16 \%)$ referrals were made by educators for vignettes written in AAVE out of 82 possible referrals versus two (2\%) referrals for vignettes written in MAE out of 82 possible referrals. Results indicate that referrals were made for vignettes written in both AAVE and MAE; however, there were more referrals made for vignettes written in AAVE than in MAE. Referrals to special education were only made by teachers and support personnel and professional roles presented with varying reasons for why they did and did not make referrals to special education. See Table 6 below.

$\begin{aligned} & \text { Table } 6 \\
& \text { Frequencies and Percentages }\end{aligned}$
\begin{tabular}{lcc} 
& \\
& Vignettes Written in & Vignettes Written in \\
& AAVE: Referred for & MAE: Referred for \\
Special Education & Special Education \\
Administrators & $0(0)$ & $0(0)$ \\
Support Personnel & $2(2)$ & $0(0)$ \\
Teachers & $4(5)$ & $1(1)$ \\
Other & $7(9)$ & $1(1)$ \\
Total Responses & $13(16)$ & $2(2)$ \\
\hline
\end{tabular}

Note: Percentages appear in parentheses.

Of the $15(9 \%)$ referrals made for special education services, general education teachers, speech-language pathologists and other are the only professional roles that made referrals to special education. General education teachers made a total of four $(5 \%)$ referral recommendations to special education after reading vignettes in AAVE and one (1\%) special 
education referral after reading vignettes in MAE. Speech-language pathologists made a total of two $(2 \%)$ recommended referrals to special education after reading vignettes in AAVE and zero $(0 \%)$ after reading vignettes in MAE. The category other made a total of seven $(9 \%)$ recommended referrals to special education after reading vignettes in AAVE and one (1\%) special education referral after reading vignettes in MAE. All other disciplines made zero ( $0 \%)$ recommended referrals for special education services after reading vignettes in AAVE and MAE.

Of the 82 responses to the vignettes written in AAVE, $13(16 \%)$ recommendations were made for a referral to special education for vignettes written in AAVE. Of the 82 responses to the vignettes written in MAE, two (2\%) recommendations were made for a special education referral for vignettes written in MAE. It is important to note that none of the vignettes presented with errors in neither AAVE nor MAE.

\section{Vignettes Written in AAVE}

Of the 82 responses to the vignettes written in AAVE, four (5\%) referrals were made by teachers. Of the four (5\%) teachers who made referrals, all were only made by general education teachers, as no referrals were made by special education classroom teachers or special education resource teachers. Of the two (2\%) referrals made by support personnel, all were only made by speech-language pathologists as no referrals were made by occupational therapists or occupational therapists who also participated in the study. Administrators made zero ( $0 \%)$ referrals for vignettes written in AAVE while seven (9\%) educators classified as other made referrals.

\section{Vignettes Written in MAE}

Of the 82 responses to the vignettes written in MAE, there were two (2\%) referrals made for a special education referral. Of those two, there was one (1\%) referral made by a general 
education teacher and one (1\%) referral made by an educator in the category other. Neither administrators nor support personnel made zero $(0 \%)$ referrals for vignettes written in MAE.

\section{Response to Vignettes by Professional Role}

Of the $15(9 \%)$ referrals made for special education services, general education teachers, speech-language pathologists and other are the only professional roles that made referrals to special education. General education teachers made a total of four $(5 \%)$ referral recommendations to special education after reading vignettes in AAVE and one (1\%) special education referral after reading vignettes in MAE. Speech-language pathologists made a total of two $(2 \%)$ recommended referrals to special education after reading vignettes in AAVE and zero $(0 \%)$ after reading vignettes in MAE. The category other made a total of seven $(9 \%)$ recommended referrals to special education after reading vignettes in AAVE and one (1\%) special education referral after reading vignettes in MAE. All other disciplines made zero (0\%) recommended referrals for special education services after reading vignettes in AAVE and MAE.

\section{Administrators}

Of the 82 responses to the vignettes written in AAVE, zero $(0 \%)$ referrals were made by administrators. Of the 82 responses to the vignettes written in MAE, there were zero $(0 \%)$ referrals made by administrators.

\section{Support Personnel}

Of the two (2\%) referrals made by support personnel, all were only made by speechlanguage pathologists and both were for vignettes written in AAVE. No referrals were made by occupational therapists or occupational therapists who also participated in the study. 


\section{Teachers}

Of the 82 responses to the vignettes written in AAVE, four $(5 \%)$ referrals were made by teachers. Of the four $(5 \%)$ teachers who made referrals, all were only made by general education teachers, as no referrals were made by special education classroom teachers or special education resource teachers. Of the 82 responses to the vignettes written in MAE, in-service teachers made one $(1 \%)$ referral for a special education referral. Of that one (1\%) referral, it was only made by a general education teacher as no referrals were made by special education classroom teachers or special education resource teachers.

\section{Other}

Educators classified as other made seven (9\%) referrals for vignettes written in AAVE and one (1\%) special education referral for a vignette written in MAE.

\section{Reason for Special Education Referrals}

To analyze the reasons educators provided for making the decision to refer or not to refer to special education, responses were coded, categorized, and themed to identify patterns (Miles et al., 2018). After I reviewed responses $(n=164)$ from the participants $(n=41)$ for each of the vignettes written in $\operatorname{AAVE}(n=2)$ and $\operatorname{MAE}(n=2)$, the responses were coded for particular words or phrases that reoccurred in the narration. However, given the small sample size, patterns are difficult to identify with certainty.

The four themes that were identified in this study included additional information needed, general education factors, perspectives on diversity, and special education process. It is important to note that codes were not mutually exclusive for each sentence; therefore, at times, sentences designated more than one code. Two of the four themes were further analyzed in greater detail to find commonalities amongst the educators for this study. The two themes which 
were assessed in greater detail for this study were perspectives on diversity and special education process.

\section{Additional Information Needed}

The theme additional information needed included the categories factors, reasons, not enough information, and time. For example, the code factors was used to highlight the following response made by a superintendent, "This answer is really dependent on the individual school." The code reasons were used for this sentence made by an educator in the category other, "We are bordering now and this answer is really dependent on the individual school." The code not enough information was used when a director of special education answered the question of why they made that decision with the following sentence, "There is not sufficient information to determine the impact on learning or interventions." An example of when the code time was used includes the following sentence stated by a general education teacher, "Without knowing time of year, interventions tried it's hard to determine."

\section{General Educational Factors}

The theme general educational factors included the categories grammar, alphabetic, negative (inaccuracy), disability, and system of support. For example, the code grammar was used for the following response made by an educator in the category other, "Everyone should be taught 'conventional' grammar in school to give them the skills and opportunity to communicate effectively in the professional world.". The code alphabetic was used for the following sentence written by a special education resource teacher, "On level writing maybe slightly above". The code topic was used for the following sentence written by an educator classified as other, "Stayed on topic for the most part". The code negative (inaccuracy) was used for the following sentence written by a general education classroom teacher, "Thoughts are jumbled in connection 
with errors." The code disability was used for the following response written by an educator classified as other, "Nothing that rises to the level of this student seeming to have a learning disability or that they are alarmingly behind.". The code system of support of was used for the following response written by a special education classroom teacher, "Student has minor mistakes that can be addressed in MTSS support groups.”.

\section{Perspectives on Diversity}

The theme perspectives on diversity included the categories diversity, dialect, and AAVE. For example, the code diversity was used for the following response by a special education classroom teacher, "This appears to be written as the student is speaking with a diverse cultural background." as well as an educator in the category other who wrote the following response, "Is this a kiddo who is ELL?". The code dialect was used for this school speech-language pathologist's following response, “This writing sample consists of dialectal features so I would be reserved in referring this student for an assessment." This following response made by a superintendent, "He has learned that way of speaking, so I imagine he is capable of learning another American dialect." was used for the code dialect as well. The code AAVE was used for this following response made by a general education classroom teacher, "It appears that this vignette is written by a speaker of AAE and would not qualify for SPED.”, and this following response by made by an educator in the category other, "He should be referred due to the writer's use of what could be called Black vernacular.". Also, this following response made by a school speech-language pathologist was coded as $A A V E$, “This seems to be written by a student who speaks African American Vernacular English. This is not an impairment.”. 


\section{Administrators}

Administrators $(n=6)$ only mentioned diversity and dialect in their responses to why they did or did not make a referral for special education services after reading vignettes in MAE and AAVE. Administrators made no specific reference to AAVE. In particular, directors of special education $(n=3)$ made references to dialect while superintendents $(n=3)$ made reference to both diversity and dialect.

\section{Support Personnel}

Support personnel $(n=8)$ listed all three themes related to diversity in their rationale for considering special education referral for students based on written vignettes. Particularly, speech-language pathologists $(n=4)$ are the only support personnel discipline who listed all three themes in their responses, including diversity, dialect, and AAVE, while case managers $(n=3)$ and occupational therapists $(n=1)$ made no reference to diversity in their responses for vignettes written in neither AAVE nor MAE. Speech-language pathologists $(n=4)$ are the only professionals of all the educators surveyed to comment on all three aspects of perspectives on diversity coded in this study: diversity, dialect, and AAVE. Also, speech-language pathologists $(n=4)$ and general education teachers $(n=7)$ are the only two educators surveyed to make mention of AAVE in particular.

\section{Teachers}

Teachers $(n=14)$ made mention of diversity and AAVE, but not dialect. Specifically, general education teachers $(n=7)$ only made reference to AAVE, special education teachers $(n=4)$ made reference to diversity, and special education resource teachers $(n=3)$ made no mention of any themes related perspective on diversity at all. It is important to note that general education 
teachers $(n=7)$ and speech-language pathologists $(n=4)$ are the only two educators surveyed to make mention of AAVE directly.

\section{Other}

Educators classified as other $(\mathrm{n}=13)$ made mention of diversity and AAVE, but not dialect.

\section{Special Education Process}

As stated, references were not analyzed as positive or negative, but merely as existence of a determining factor during the decision-making process for referral to special education. For example, the code special education was used for the following sentence stated by a general education classroom teacher, "I do see students referred to SPED all too often.". The code proposal was used for the following sentence stated by an educator categorized as other, "I would recommend interventions as part of the pre referral process.". The code treatment was used for the following sentence made by an educator categorized as other, "The student demonstrates a need for reteaching/intervention.". The code assistance was used for the following sentences such as, "He just needs some help." and "We can work on this. Unfortunately, in a more affluent district, we might put this student on a watch list.", made by a general education classroom teacher and an educator classified as other, respectively.

\section{Administrators}

Administrators $(n=6)$ made mention of all four codes related to the theme of special education process as a reason for sending or not sending a child for a special education referral after reading vignettes in MAE and AAVE. The four categories included special education, proposal, treatment, and assistance. However, directors of special education $(n=3)$ made references to all four themes, whereas superintendents $(n=3)$ made reference to none. 


\section{Support Personnel}

Support personnel $(n=8)$ mentioned of all four codes related to the theme of special education process as a reason for sending or not sending a child for a special education referral after reading vignettes in MAE and AAVE. The four categories included special education, proposal, treatment, and assistance. Particularly, case managers $(n=4)$ made references to all four themes, occupational therapists $(\mathrm{n}=1)$ made reference to only treatment, and speechlanguage pathologists $(\mathrm{n}=4)$ made reference to none.

\section{Teachers}

Teachers ( $\mathrm{n}=14)$ made mention of all four codes related to the theme of special education process as a reason for sending or not sending a child for a special education referral after reading vignettes in MAE and AAVE. The four categories included special education, proposal, treatment, and assistance. Specifically, all in-service teachers surveyed $(n=14)$ referenced all four codes related to the theme of special education process, including general education teachers $(n=7)$, special education teachers $(n=4)$, and special education resource teachers $(n=3)$. Teachers is the only category where all disciplines surveyed made reference to all coded themes.

\section{Other}

Educators classified as other $(\mathrm{n}=13)$ made mention of all four codes related to the theme of special education process, including special education, proposal, treatment, and assistance. 


\section{CHAPTER V: DISCUSSION}

\section{Overview of the Study}

This study used mixed method concurrent design methodology (Creswell et al., 2017). The purpose of this research study was to investigate the effects of third graders' vignettes written in AVVE on educators' referral process to special education. For this study, in-service educators completed an online 25-item survey based on the work of Champion et al., (2012). The survey used a 6-point Likert scale and was designed to assess educators' general attitude toward AAVE and their perceptions of AAVE as a legitimate dialect for use in an academic setting.

In-service educators also read two vignettes written in AAVE and two vignettes written in MAE and made decisions about referring the $3^{\text {rd }}$ graders for a special education referral based on their writing samples. Written vignettes were based on the works of Common Core State Standards Initiative (2010), Fogel and Ehri (2000), and Reading Rockets (2020). Educators then explained why they made their decision to refer or not to refer for a special education evaluation using a word, phrase, or sentence.

Demographic information was collected via questionnaire with in-service educator respondents presenting with various levels of exposure to students who speak AAVE. Total inservice educators $(\mathrm{n}=41)$ who responded included administrators $(\mathrm{n}=6)$, support personnel $(n=8)$, teachers $(n=14)$, and category other $(n=13)$. Administrators referred to directors of

special education $(n=3)$ and superintendents $(n=3)$; support personnel referred to case managers $(n=3)$ occupational therapists $(n=1)$, and speech-language pathologists $(n=4)$; and teachers referred to general education classroom teachers $(n=7)$, special education classroom teachers $(n=3)$, and special education resource teachers $(n=4)$. The research questions for this study were: 
1. What are the perceptions of in-service teachers, support personnel, and/or administrators towards the AAVE dialect?

2. How do in-service teachers, support personnel, and/or administrators evaluate texts written in AAVE versus MAE as related to special education referral?

\section{Interpretation of Survey Results}

When in-service educators were asked to rate their perceptions of AAVE in the online survey (Champion et al., 2012), teachers, support personnel, and administrators presented with mixed results regarding their general attitudes towards AAVE based on mean results from the 25-question survey. Administrators presented, support personnel, and teachers overall presented with positive general attitudes towards AAVE based on mean scores derived from the survey on itemized questions. Also, all in-service educators presented with overall positive general perceptions of AAVE as a legitimate dialect and supported its use in the classroom setting based on mean scores derived from the survey. These findings are in direct contrast to previous research findings which indicated that teachers and related service providers have negative perceptions about using the dialect at school (Gupta, 2010; Newkirk-Turner, Williams, Harris, \& McDaniels, 2013).

In particular, participants presented with attitudes that AAVE is a legitimate dialect that may boost pride amongst African Americans and that eliminating AAVE is damaging to African Americans. Also, all educators agreed that use of AAVE motivates academic achievement for African Americans, should be allowed in classrooms, and should be taught in schools. All educators rejected the notion that schools should standardize the English language and they all agreed that the rejection of native language is harmful. All educators expressed attitudes that AAVE does not have faulty grammar or sound sloppy. However, although administrators 
expressed feelings that AAVE is cool or remarkable, support personnel and teachers did not express this sentiment. This differential may be significant as support personnel and teachers are the only named professionals who made special education referrals for vignettes written in AAVE in this study.

Findings in this present study were different from findings in previous studies. In previous studies, researchers found that educators expressed global negative perceptions of AAVE as a dialect and negative perceptions of the use of AAVE at school overall (Champion et al., 2012; Gupta, 2010; Newkirk-Turner et al., 2013). However, it is important to note that the survey used in this present research study is based on the work of Champion et al. (2012), which is not the same survey used in the studies completed by Gupta (2010) and Newkirk-Turner et al. (2013). Gupta (2010) and Newkirk-Turner et al. (2013) utilized the same survey, whereas Champion et al., (2012) used a different one. Therefore, comparisons of results from this current study based on the survey work of Champion et al., (2012) to the survey work of Gupta (2010) and Newkirk-Turner et al. (2013) should be done with caution. The survey used in Gupta (2010) and Newkirk-Turner et al. (2013) reflected teachers' perceptions of AAVE, and preparedness to address teaching speakers of AAVE.

Also, when making comparisons to survey results found by Champion et al., (2012) to this present study which used the same survey, it is important to note that the demographics in the Champion et al. (2012) study differ greatly from those found in this present study. For example, the Champion et al., (2012) study was comprised exclusively of undergraduate, preservice education majors, all of whom were enrolled in an undergraduate course titled "Teaching Diverse Populations". Further, participants in the Champion et al., (2012) course took the survey on the first day of their class titled "Teaching Diverse Populations". This means the survey was 
completed before full exposure to the topic of teaching diverse populations. Therefore, although the survey in the Champion et al., (2012) study is the same as the instrument used in this present study, the participants and procedures differ so widely between the two studies that direct comparison of results should be done with hesitancy.

That said, based on the results from the survey in this study, it is possible that strides are being made amongst in-service educators to openly address and acknowledge a relationship amongst explicit bias against African Americans, the dialect AAVE, and the use of AAVE in the classroom setting (Amodio et al., 2010). As stated, given the documented patterns of overrepresentation of African American students in special education in the United States, the process of building cultural competence may well begin with recognition and appreciation of African American cultural linguistic differences in AAVE (Blanchett, 2006; DeJarnette et al., 2015; Harry et al., 2014; Rivers et al., 2012). Educators that present with an increased awareness of cultural bias and an ability to explicitly discriminate between cultural difference and deficits may assist staff in better assessing African American students so that disproportionality in special education does not continue to persist within school districts (Skiba, Simmons, et al., 2006).

\section{Interpretation of Written Vignettes Results}

When in-service educators were asked to read $3^{\text {rd }}$ grade vignettes written in AAVE and MAE and make decisions about special education referrals, a total of $13(16 \%)$ of the vignettes written in AAVE were referred for a special education evaluation while $2(2 \%)$ of vignettes written in MAE were referred for a special education evaluation. Support personnel made two (2\%) referrals for texts written in AAVE out of 82 total responses and $0(0 \%)$ for texts written in

MAE out of 82 total responses. Teachers made four (5\%) referrals for texts written in AAVE out 
of 82 total responses and one (1\%) written in MAE out of 82 total responses. The category other made seven $(9 \%)$ referrals for texts written in AAVE out of 82 total responses and one (1\%) referral for texts written in MAE out of 82 total responses. Administrators made zero ( $0 \%)$ referrals for special education services for texts written in AAVE out of 82 total responses and zero $(0 \%)$ referrals for texts written in MAE out of 82 total responses.

Results from this study suggest that vignettes written in AAVE were referred for evaluation 6.5 times more frequently than vignettes written in MAE. However, given the small sample size of 41 participants who responded to survey items in this study, it is problematic to draw comprehensive, generalized conclusions based on this small sample. Overall, the findings in this study are consistent with previous research findings that African American students are two to three times more likely to be over-identified than their White same-age peers in special education within disability categories that are subjective in nature, lack clear definitions, and rely on clinical judgement, such as specific learning disability (Bal et al., 2014; NCES, 2016). However, it is important to note that results of the Bal et al., (2014) study are based on a mixedmethod collaborative case analysis that examined local patterns of disproportionality in an urban school using archival data from 2006 through 2010, while this current study uses a mixed method concurrent design (Creswell et al., 2017). Therefore, it may be problematic to make direct comparisons from the findings in the Bal et al., (2014) study to the findings in this current study.

Of the referrals made to special education, it is interesting to note that speech-language pathologists $(n=4)$ and general education teachers $(n=7)$ were the only two named professional disciplines who made referrals to special education. The category other $(n=13)$ also made 
referrals to special education after reading the vignettes. Administrators $(n=6)$ are the only professional category who made no referrals for special education services during this study.

General classroom teachers and speech-language pathologists were the only participants who identified vignettes written in AAVE for a special education evaluation in this study. Skiba, Simmons et al. (2006) found in their study that administrators and teachers believed that African American students were over-referred to special education, possibly due to a cultural mismatch or perhaps insufficient training. Moreover, Skiba, Simmons et al. (2006) found that administrators and teachers reported it problematic that oftentimes the sole resource available for students with individual academic needs was special education services. However, Skiba, Simmons et al. (2006) found that it was only administrators who reported that this was a phenomenon that probably increased the number of inappropriate referrals to special education. That said, given the small sample size of 41 participants who responded to survey items in this study, only six of which were administrators, it is difficult to make sweeping assumptions from this small sample size.

When educators were asked to explain why they did or did not recommend special education services after reading vignettes written in AAVE and MAE, themes emerged which were consistent with those identified and described by Skiba, Simmons et al. (2006). Those themes were additional information needed, general education factors, perspectives on diversity, and special education process. Of those four themes identified in this study, I explored two of the four themes in greater detail: perspectives on diversity and special education process.

\section{Perspectives on Diversity}

Skiba, Simmons et al. (2006) described the theme perspectives on diversity as the problematic overuse of special education for students of color used by teachers when faced with 
academic or social problems among the students in their classrooms. Given that description, a referral to special education is used by teachers as the primary method to offer additional resources for students who are struggling. Further, participants in Skiba, Simmons et al. study (2006) openly recognized that overrepresentation of minority students in special education was a serious problem, yet they also conveyed that their rationale for referral to special education was to help the student succeed.

Based on Skiba, Simmons et al.'s (2006) description of perspectives on diversity, I found similar thematic patterns in this study and applied the same theme to describe respondents' response. For example, the only three categorical professionals who made referrals to special education in this study were general education classroom teachers, speech-language pathologists, and category other. These three professional roles were also the only ones who directly referred to AAVE by name, openly recognizing and acknowledging the presence of this specific linguistic dialectal difference. None of the other professionals' roles made reference to AAVE directly, although they discussed dialect and diversity in general. For example, one teacher wrote, "He should be referred due to the writer's use of what could be called Black vernacular.”. This is an example of an educator openly recognizing the dialectal difference spoken by the student in AAVE, but still referring the student for special education as if it were a disorder.

\section{Special Education Process}

I also explored the theme special education process in greater details for this study. Based on Skiba, Simmons, et al.'s (2006) description of the theme special education process, the term is focused on minority disproportionality in special education and the possible cause of it being the direct contribution of the referral, assessment, and decision-making process that 
ultimately leads to eligibility for special education. In other words, it was described to mean that the referral-to-placement process alone contributes to minority disproportionality in itself (Skiba, Simmons, et al., 2006). The theme special education process was further described to include the lack of resources for classroom teachers as a likely contributor to racial disparities in referral and placement (Skiba, Simmons, et al., 2006).

Based on Skiba and Simmons et al.'s (2006) description of special education process, I found similar thematic patterns in this study and applied the same theme to describe respondents' response. In fact, all four categorical professional roles, administrators, teachers, support personnel, and category other made reference to the special education process within their responses to explain their decision-making process. This seems consistent with Skiba et al.'s (2006) claim that the special education process itself is viewed by educators as a mere extension of an existing process that educators use when faced with a lack of resources in the classroom. In other words, the response when an educator lacks resources to help a student, the response is to refer to special education (Skiba, Simmons, et al., 2006). I found these same themes in this study and coded it under the theme of special education process. For example, one educator in this study wrote, "I do see students referred to SPED all too often.”, and another wrote, "I would recommend interventions as part of the pre referral process.”, and yet another wrote, "Unfortunately, in a more affluent district, we might put this student on a watch list.".

Also, although participants in this study supported the use of AAVE in the classroom setting based on mean scores derived from the survey based on the survey (Champion et al., 2012), referral rates were 6.5 times greater for AAVE writers than MAE writers. This seems consistent with Skiba et al.'s (2006) claim that the special education process itself is viewed by educators as a mere extension of an existing process when educators lack resources in the 
classroom. In other words, it is possible that when faced with writings outside of MAE, educators do not know how to respond apart from the special education process. These findings further highlight the research study completed by Skiba et al. (2006), where it was concluded that the referral process that perpetuates disproportionality in special education is complex and that there is need to increase educational resources for African American students.

The findings from this study seem consistent with Skiba et al.'s (2006) regarding emerging themes related to perspectives on diversity and special education process. However, correlations to this current study and Skiba et al.'s (2006) study should be met with temperance given the vast differences in sample size and methodology. Skiba et al.'s (2006) study consisted of 66 educators interviewed in a confidential manner, face-to-face, in focus groups, and across several school districts. The semi-structured interviews were specifically designed to discuss their views on minority disproportionality in special education related to the school district where they worked. This study was completed online using a survey design and consisted of 41 anonymous education participants. Although similarities are present, direct comparisons should be made with caution due to differences in the study designs and sample size.

\section{Speech-Language Pathologists}

Speech-language pathologists were the only discipline which listed all three themes related to perspectives on diversity in their responses. In other words, speech-language pathologist included diversity, dialect, and AAVE in their responses when considering a referral for special education. These findings are consistent with ASHA's (2018) reinforcement of cultural competence in Speech-Language Pathology training that teaches self-awareness about the languages, dialects, and cultures of others, as well as their own through self-analysis to assess cultural biases and improve self-awareness (ASHA, 2018). However, it is possible that speech- 
language pathologist may lack awareness of the specific rules and aspects related to various cultural and linguistic differences. This is a crucial aspect when evaluating students who speak AAVE that may be over-looked when training Speech-Language Pathologists so they do not draw conclusions regarding disorder versus dialectal differences based on norms that may be irrelevant to the student (Beneke et al., 2015; Craig et al., 2014; Oetting, 2018).

\section{Limitations}

It is important to highlight the limitations to this study and its impact on the interpretation of results. As stated, the small sample size of 41 respondents is an obvious limitation of this study. It is possible that the low sample size may have had been a direct result of educators' limited voluntary responses to requests to complete the presented survey via direct email and social media. This small sample size has a direct impact on the reliability and generalizability of the survey results in this study as a small sample size is less representative of the entire population.

Also, participants were allowed to identify themselves as belonging to a particular demographic category and self-report their responses on the survey. Although the survey study was anonymous, it is still possible that respondents may have edited their responses to match responses they believed may be more socially acceptable. Therefore, it is possible that questions related to attitudes and perceptions of AAVE may be skewed.

Another limitation is that vignettes were only written as $3^{\text {rd }}$ grade samples. It is possible that different results may be derived using a different age-range sample. Likewise, not all educators who completed the survey were $3^{\text {rd }}$ grade educators. It is possible that educators who evaluated the samples may have had a differing positive or negative perception based on the educators' own experiences with the $3^{\text {rd }}$ grade age group. 
Also, a limitation is within the written vignettes themselves as that the samples did not provide any additional information regarding the educational status of the student. Additional information might have included if or how long the student had been presenting with difficulties in class, how much time the student had been given to write the sample, whether or not the student had ever received interventions related to writing, and whether the student presented with any previously identified diagnoses or disabilities. It is possible that if more information such as these had been provided, with additional context related to the students who wrote the samples, educators' responses and decisions to refer to special education after reading the written vignettes may have differed based on the educators' receiving increased information on the subject matter. In other words, the limited information provided in the vignettes makes the implied presumption that the educator participants are already fully aware of the subject matter of writing abilities and are able to successfully evaluate the literacy skills of a $3^{\text {rd }}$ grader.

Additionally, a limitation to this study includes that reverse-coding variables were not used to analyze data for participants' mean scores and standard deviations of educators' attitudes towards AAVE by category. Since this analysis was not completed, it is difficult to make overall assumptions about group means. Reverse-coding may allow for a more accurate analysis of responses on every survey item and improve validation of results.

\section{Future Research}

There is need to further analyze the categories of professionals who made referrals to special education in this study. This information may be an important component when developing training programs for administrators, teachers, and support personnel in the future. Likewise, analysis of educators' responses to AAVE versus MAE by gender, race, level of 
education, and years working in education may all be valuable information when considering future research on bias against AAVE and its impact on disproportionality in special education.

Also, future research might include written vignettes in MAE versus AAVE that is paired with background information regarding the context of the student. This may serve to expand on identified themes to explain why educators make referrals for special education. Further, presenting written vignettes in age ranges other than $3^{\text {rd }}$ grade may produce differing substantial results. Also, providing participants with detailed information regarding expectations for a written vignette in a presented age group may prove beneficial to study differences in responses to vignettes as well.

Further, as speech-language pathologists and general education classroom teachers were the only two named disciplines in the study who made referrals for special education in this study, research that explores comparisons and contrasts between these two disciplines may be warranted. Moreover, gaining greater insight into why directors of special education did not make referrals to special education after reading any of the vignettes should be further explored. That said, it may be interesting to compare and contrast the training and professional development of all educators represented in this study, including administrators, support personnel, and teachers, to analyze for patterns between training and referral rate to special education.

Similarly, future research might include face-to-face interviews with educators to assess their perception of AAVE and its potential use in the classroom setting within instruction (Champion et al., 2012). Semi-structured interviews might include questions designed to gain insight into educators' perceived impact of the process that may contribute to special education disproportionality for African Americans (Skiba, Simmons, et al., 2006). 
Also, culturally responsive pedagogy is named by leaders in the field as one of the most effective instructional tools designed to meet the learning needs of CLD students (Gay, 2002, 2010; Harmon, 2012; Ladson-Billings, 1995, 2000, 2006). There continues to be a need to find ways to include African Americans who speak AAVE in the culturally responsive pedagogy in schools in research. Analyzing data responses to AAVE based on level of education related cultural linguistic difference may one way to assess this impact.

Lastly, implicit and explicit bias against AAVE and its relationship to disproportionality in special education is a complex issue in need of further study. Several factors to consider when assessing this bias through research in the future include the cultural linguistic mismatch between teachers and students, the subjective process for referrals, biased perception of cultural differences, and negative views of those who use the language system of AAVE to communicate (McKenna, 2013).

\section{Conclusion}

Given the United States public school's documented patterns of overrepresentation of African American students in special education, the process of building cultural competence to implement effective pedagogy may well begin with recognition and appreciation of African American cultural linguistic differences in AAVE (Blanchett, 2006; DeJarnette et al., 2015; Harry et al., 2014; Rivers et al., 2012). This may help offer educators tools to be better aware of cultural bias and the need to explicitly discriminate between cultural difference and deficits based on disability. Further, it may also assist staff in better assessing African American students' intellectual and behavioral abilities so that disproportionality in special education does not continue to persist within the school district (Skiba, Simmons, et al., 2006). 
With the United States public schools presenting with greater diversity in student population, there is need to ensure equitable and valuable school experiences for all students represented. In fulfilling this need, educators can increase awareness and responsiveness to students' cultures and the contexts in which they are situated (Fallon, O’ Keeffe, \& Sugai, 2012). In addition to building cultural competence, educators can gain knowledge about cultural values and patterns to aid special education team members to appropriately circumvent mistaken referrals that stem from misperceived differences as disabling conditions (Collier, 2010).

Exploration of developmental norms in AAVE, as well as examinations about teaching and learning of AAVE speakers, will help propel cultural consideration within the education paradigm as it will urge educators to use their own knowledge of the complex views of culture and language. Likewise, research methodologies can explore insights into cultural linguistics using a systematic and interdisciplinary approach. This will allow for improved understanding of inclusive, equitable education, educational system accountability, and opportunities for students to learn through the overall RtI, MTSS, and PBIS systems. An educational approach that includes culture will allow for educator humility, and the necessity of representing communities and their cultural linguistic values using everyday practices consistent with the students' culture, instead of overwriting them (Sullivan, Artiles, Hernandez-Saca, 2015). 


\section{REFERENCES}

Alim, H. S. (2005). Critical language awareness in the United States: Revisiting issues and revising pedagogies in a resegregated society. Educational Researcher, 34(7), 24-31.

Alim, H. S., Rickford, J. R., \& Ball, A. (Eds.). (2016). Raciolinguistics: How language shapes our ideas about race. New York, NY: Oxford University Press.

Amberg, J. S., \& Vause, D. J. (2009). American English: History, structure, and usage. New York, NY: Cambridge University Press.

Amodio, D. M., \& Mendoza, S. A. (2010). Implicit intergroup bias: Cognitive, affective, and motivational underpinnings. In B. Cawronski \& B. K. Payne \{Eds.), Handbook of implicit social cognition: Measurement, theory, and applications (pp. 353-374). New York: Cuilford Press, 2010.

Annamma, S. A., Connor, D., \& Ferri, B. (2013). Dis/ability critical race studies (DisCrit): Theorizing at the intersections of race and dis/ability. Race Ethnicity and Education, 16, 1-31.

Artiles, A. J. (2015). Beyond responsiveness to identity badges: Future research on culture in disability and implicatinos for response to intervention. Educational Review, 67(1), 1-22.

Artiles, A. J., \& Kozleski, E. B. (2007). Beyond convictions: Interrogating culture, history, and power in inclusive education. Language Arts, 84, 357-360.

Artiles, A. J., Kozleski, E. B., \& Waitoller, F. R. (Eds.). (2011). Inclusive education: Examining equity on five continents. Cambridge, MA: Harvard University.

Artiles, A. J., Kozleski, E. B., Trent, S. C., Osher, D., \& Ortiz, A. (2010). Justifying and explaining disproportionality, 1968-2008: A critique of underlying views of culture. Exceptional Children, 76(3), 279-299. 
ASHA (American Speech-Language-Hearing-Association). (2018). Cultural competence: Key issues. PracticePORTAL. Retrieved from https://www.asha.org/PRPSpecificTopic.aspx?folderid=8589935230\&section=Key_Issue S

Bal, A., Sullivan, A., \& Harper, J. (2014). A situated analysis of special education disproportionality for systemic change in an urban school district. Remedial and Special Education, 35, 3-14. doi:10.1177/0741932513507754

Beneke, M. \& Cheatham, G. A. (2015). Speaking up for African American English: Equity and inclusion in early childhood settings. Early Childhood Education Journal, 43, 127-134. doi 10.1007/s10643-014-0641-x

*Blackburn, J. F. (2012). The effect of dialect instruction on student knowledge of and attitudes toward African American English. Communications Disorders Quarterly, 33(4), 220229.

Blake, R. \& Cutler, C. (2003). AAE and variation in teachers' attitudes: A question of school philosophy? Linguistics and Education, 14, 163-194.

Blanchett, W. J. (2006). Disproportionate representation of African American students in special education: Acknowledging the role of white privilege and racism. Educational Researcher, 35(6), 24-28.

Bliss, L. S., \& McCabe, A. (2008). Personal narratives: Cultural differences and clinical implications. Topics in Language Disorders, 28(2), 162-177.

Brown, B. A. (2006). It isn't no slang that can be said about this stuff: Language, identity, and appropriating science discourse. Journal of Research in Science Teaching, 43(1), 96-126.

Brown v. Board of Education, 347 United States 483 (1954). 
Campbell, E. E. \& Nicol, J. J. (2017). African American English And urban literature: Creating culturally caring classrooms. \#CritEdPol: Journal of Critical Education Policy Studies at Swarthmore College: 2(1), 1-19.

Cazden, C. B. (1981). Language in early childhood education. Washington, DC: National Association for the Education of Young Children.

*Champion, T., Cobb-Roberts, D., Bland-Steward, L. (2012) Future educators' perceptions of African American Vernacular English (AAVE). Online Journal of Education Research, $5(1), 80-89$.

Clark, E. V. (2007). Conventionality and contrast in language and language acquisition. New Directions for Child and Adolescent Development, 115, 11-23.

Collier, C. (2010). Seven steps to separating difference from disability. Thousand Oaks, CA: Corwin Press.

Common Core State Standards Initiative. (2010). Common core state standards for English language arts and literacy in history/social studies, science, and technical subjects. Washington, DC: Council of Chief State School Officers and National Governors Association. Retrieved from http://www.corestandards.org/

Council for Children with Behavioral Disorder (CCBD): A Division of the Council for Exceptional Children. (2013). CCBD'S position summary on federal policy on disproportionality in special education. Behavioral Disorders, 38, 108-12.

Craig, H. K., Kolenic, G. E., \& Hensel, S. L. (2014). African American English-speaking students: A longitudinal examination of style shifting from kindergarten through second grade. Journal of Speech, Language, and Hearing Research, 57, 143-157. 
Craig, H. K., Zhang, L., Hensel, S. L., \& Quinn, E. J. (2009). African American English speaking students: An ex- amination of the relationship between dialect shifting and reading outcomes. Journal of Speech, Language, and Hearing Research, 52, 839-855.

Creese, A. \& Blackledge, A. (2010). Translanguaging in the bilingual classroom: A pedagogy for learning and teaching. Modern Language Journal, 94, 103-115.

Creswell, J. W., \& Creswell, J. D. (2017). Research design: Qualitative, quantitative, and mixed methods approaches. Sage publications.

DeJarnette, G., Rivers, K. O., \& Hyter, Y. D. (2015). Ways of examining speech acts in young African American children: Outside-in versus inside-out. Topics in Language Disorders, $35,59-73$.

Delpit, L. (2006). Other people's children: Cultural conflict in the classroom. New York: New Press.

Delpit, L. (2012). What should teachers do? Ebonics and culturally responsive instruction. In S. J. Nero (Ed.), Dialects, Englishes, creoles, and education (pp. 93-101). Mahwah, NJ: Lawrence Erlbaum Associates.

Dever, B. V., Raines, T.C., Dowdy, E., \& Hostutler, C. (2016). Addressing disproportionality in SED using a universal screening approach. Journal of Negro Education, 85(1), 59-71.

Dyson, A. H., \& Smitherman, G. (2009). The right (write) start: African American language and the discourse of sounding right. The Teachers College Record, 111(4), 973-998.

Fallon, L. M., O’Keeffe, B. V., \& Sugai, G. (2012). Consideration of culture and context in school-wide positive behavior support: A review of current literature. Journal of Positive Behavior Interventions 14(4), 209-219. doi: 10.1177/1098300712442242 
Ferguson, R. F. (2007). Toward excellence with equity: An emerging vision for closing the achievement gap. Boston, MA: Harvard Education Press.

Flores, N., \& Rosa, J. (2015). Undoing appropriateness: Raciolinguistic ideologies and language diversity in education. Harvard Educational Review, 85(2), 149-171.

*Fogel, H., \& Ehri, L. C. (2006). Teaching African American English forms to standard American English-speaking teachers. Journal of Teacher Education, 57, 464-480.

Fogel, H., \& Ehri, L. C. (2000). Teaching elementary students who speak Black English Vernacular to write in Standard English: Effects of dialect transformation practice. Contemporary Educational Psychology, 25(2), 212-235.

Ford, D. Y. (2012). Culturally different students in special education: Looking backward to move forward. Exceptional Children, 78(4), 391-405.

Ford, D. Y., \& Toldson, I. A. (2015, July 5). Study on Black, Hispanic children in special ed wrong, regressive. Diverse Issues in Higher Education. Retrieved from http://diverseeducation.com/article/76088

Ford, J. F. (1978). The prospective foreign language teacher and the culturally and linguistically different learner. Foreign language annals, 11(4), 381-390.

Gatlin, B., Wanzek, J., \& Al Otaiba, S. (2016) An examination of kindergarten oral language for African American students: Are there meaningful differences in comparison to peers?, Reading \& Writing Quarterly, 32(5), 477-498, doi:

$10.1080 / 10573569.2015 .1039737$

Gay, G. (2002). Preparing for culturally responsive teaching. Journal of Teacher Education, 53, $106-116$. 
Gay, G. (2010). Culturally responsive teaching: Theory, research, and practice. New York, NY: Teachers College Press.

Godley, A. J., Sweetland, J. Wheeler, R. S., Minnici, A., \& Carpenter, B. D. (2006). Preparing teachers for dialectally diverse classrooms. Educational Researcher, 35(8), 30-37.

Green, L. (2002). African American English. Cambridge, England: Cambridge University Press.

Green, L. (2003). Syntactic and semantic patterns in child African American English. Texas

*Gupta, A. (2010). African American English: Teacher beliefs, teacher needs and teacher preparation programs. Reading Matrix: An International Online Journal, 10(2), 152-164.

Harmon, D. (2012). Culturally responsive reaching though a historical lens: Will history repeat itself? Interdisciplinary Journal of Teaching and Learning, 2(1), 12-22.

Harry, B., \& Klingner, J. K. (2014). Why are so many minority students in special education? Understanding race and disability in schools. New York, NY: Teachers College.

Hernandez, D. J. (2011). Double jeopardy: How third-grade reading skills and poverty influence high school graduation. Annie E. Casey Foundation.

Herzik, L. (2015). A better IDEIA: Implementing a nationwide definition for significant disproportionality to combat overrepresentation of minority students in special education. San Diego Law Review, 152, 951-966.

Hill, K. D. (2009). Code-switching pedagogies and African American student voices: Acceptance and resistance. Journal of Adolescent \& Adult Literacy, 53(2), 120-131. doi:10.1598/JAAL.53.2.3

Hwa-Froelich, D., Kasambira, D. C., \& Moleski, A. M. (2007). Communicative functions of African American Head Start children. Communication Disorders Quarterly, 28(2), 7791. 
Hycner, R. H. (1985). Some guidelines for the phenomenological analysis of interview data. Human studies, 8(3), 279-303.

Hyter, Y. D. (2007). Pragmatic language assessment: A pragmatics-as-social practice model. Topics in Language Disorders, 27(2), 128-145.

Hyter, Y. D., Rivers, K. O., \& DeJarnette, G. (2015). Pragmatic language of African American children and adolescents: A systematic synthesis of the literature. Top Language Disorders, 35(1), 8-45.

Individuals with Disabilities Education Act (IDEA) 2004, 34 CFR $\$ 300.646$.

Individuals with Disabilities Education Act (IDEA) 2004, 20 USC § 1416(a)(3)(C)

Labov, W. (1969a). Contraction, deletion, and inherent variability of the English copula. Language, (45), 715-762.

Labov, W. (1969b). The logic of non-standard English. In Georgetown University Monographs in Languages and Linguistics, (22).

Labov, W. (1967). Some sources of reading problems for Negro speakers of nonstandard English. In A. Frazier (Ed.), New directions in elementary English. Champaign, IL.: National Council of Teachers of English.

Labov, W. (2001). The anatomy of style-shifting. In Penelope Eckert and John R. Rickford (eds.) Style and Sociolinguistic Variation. Cambridge, U.K.: Cambridge University Press. $85-108$.

Ladson-Billings, G. (1995). But that's just good teaching! The case for culturally relevant pedagogy. Theory into Practice, 34, 159-165. 
Ladson-Billings, G. (2000). Racialized discourses and ethnic epistemologies. In N. Denzin \& Y. S. Lincoln (Eds.), The Sage handbook of qualitative research (2nd ed., pp. 257-277). Thousand Oaks, CA: Sage.

Ladson-Billings, G. (2006). From the achievement gap to the education debt: Understanding achievement in U.S. schools. Educational Researcher, 35(7), 3-12.

Lesnick, J., Goerge, R., Smithgall, C., \& Gwynne, J. (2010). Reading on grade level in third grade: How is it related to high school performance and college enrollment. Chicago, IL: Chapin Hall at the University of Chicago, 1, 12.

Lobeck, A., (2019). Teaching linguistic diversity as the rule rather than the exception. In M. D. Devereaux \& C. C. Palmer (Eds.), Teaching Language Variation in the Classroom: Strategies and Models from Teachers and Linguists. (pp. 76-83), New York, NY: Routledge Taylor \& Francis Group.

Lustig, M. W., \& Koester, J. (2012). Intercultural competence (7th ed.). Boston, MA: Pearson. Lynn, M. \& Adams, M. (2002) Introductory Overview to the Special Issue Critical Race Theory and Education: Recent Developments in the Field, Equity \& Excellence in Education, (35)2, 87-92. doi: $10.1080 / 713845285$

McKenna, J. (2013). The disproportionate representation of African Americans in programs for students with emotional and behavioral disorders. Preventing School Failure, 57(4), 206-211. doi:10.1080/1045988X.2012.687792

Merriam, S. B., \& Tisdell, E. J. (2015). Qualitative research: A guide to design and implementation. San Francisco: Jossey Bass.

Met, M. (2008). Paying attention to language: Literacy, language and academic achievement. In Pathways to Multilingualism (pp. 49-70). Multilingual Matters. 
Meyer, C. F. (2009). Introducing English linguistics. New York, NY: Cambridge University Press.

Microsoft Corporation. (2018). Microsoft Excel. Retrieved from https://office.microsoft.com/excel.

Miles, M. B., Huberman, A. M., \& Saldaña, J. (2018). Qualitative data analysis: A methods sourcebook. Sage publications.

Mordaunt, O. G. (2011). Bidialectalism in the classroom: The case of African American English. Language, Culture and Curriculum, 24(1), 77-87.

National Center for Education Statistics. (2016). Status and Trends in the Education of Racial and Ethnic Groups 2016. United States Department of Education. Retrieved from https://nces.ed.gov/pubs2016/2016007.pdf

Newkirk-Turner, B., Oetting, J., \& Stockman, I (2014). BE, DO, and modal auxiliaries of threeyear-old African American English speakers. Journal of Speech, Language, and Hearing Research, 57, 1383-1393.

*Newkirk-Turner, B. L., Williams, M., Harris, T., \& McDaniels, P. (2013). Pre-service teachers' attitudes toward students' use of African American English. Researcher: An Interdisciplinary Journal, 26(2), 41-57.

Oetting, J. B. (2018). Prologue: Toward accurate identification of developmental language disorder within linguistically diverse schools. Language, Speech, and Hearing Services in Schools, 49, 213-217.

Oetting, J. B., Newkirk, B. L., Hartfield, L. R., Wynn, C. G., Pruitt, S. L., \& Garrity, A. W. (2010). Index of productive syntax for children who speak African American English. Language, Speech, and Hearing Services in Schools, 41, 328-339. 
Patton, M. Q. (2015). Qualitative research \& evaluation methods. Los Angeles: SAGE Publications, Inc.

Qualtrics Research Suite. (2020) Qualtrics XM. Qualtrics, Provo, UT, USA. http://www.qualtrics.com

Reading Rockets. (2020). Looking at writing: Third grade writing sample 5. Colorín, Colorado: WETA Public Broadcasting. Retrieved from https://www.readingrockets.org/looking-atwriting/third-grade-writing-sample-5

Reading Rockets. (2020). Looking at writing: Third grade writing sample 4. Colorín, Colorado: WETA Public Broadcasting. Retrieved from https://www.readingrockets.org/looking-atwriting/third-grade-writing-sample-4

Renn, J. (2010). Acquiring style: The development of dialect shifting among African American children. Unpublished doctoral dissertation, University of North Carolina, Chapel Hill, NC.

Renn, J., \& Terry, J. M. (2009). Operationalizing style: Quantifying the use of style shift in the speech of African American adolescents. American Speech, 84(4), 367-390.

Roy, J., Oetting, J. B., \& Moland, C. W. (2013). Linguistic constraints on children's overt marking of BE by dialect and age. Journal of Speech, Language, and Hearing Research, $56,933-944$.

Rickford, J. R. (2019). Variation, Versatility and Change in Sociolinguistics and Creole Studies. New York, NY: Cambridge University Press

Rickford, J. R. (2016). Labov's contributions to the study of African American Vernacular English: Pursuing linguistic and social equity. Journal of Sociolinguistics, 20(4), 561580. 
Rickford, J. R., Duncan, G. J., Gennetian, L. A., Gou, R. Y., Greene, R., Katz, L. F., Kessler, R. C., Kling, J. R., Sanbonmatsu, L., Sanchez-Ordonez, A. E. (2015, September 22). Neighborhood effects on use of African American Vernacular English. Proceedings of the National Academy of Sciences of the United States of America, 112(38), 1181711822.

Rickford, J. R. (1999). Phonological and grammatical features of African American Vernacular English (AAVE), in African American Vernacular English, J. R. Rickford, Ed. Malden, MA: Blackwell, pp. 3-14.

Rivers, K.O., Hyter, Y., \& DeJarnette, G. (2012). Parsing pragmatics. ASHA Leader, 17(13), 1417.

Seltzer, K., \& de los Ríos, C. V. (2018). Translating theory to practice: Exploring teachers’ raciolinguistic literacies in secondary English classrooms. English Education, 51(1), 4979.

*Shepherd, M. A. (2011). Effects of ethnicity and gender on teachers' evaluation of students' spoken responses. Urban Education, 46(5), 1011-1028.

Skiba, R. J., Aritles, A. J., Kozleski, E. B., Losen, D. J., \& Harry, E. G. (2016). Risks and consequences of oversimplifying educational inequities: A response to Morgan et al. (2015). Educational Researcher, 45, 221-225. doi:10.3102/0013189X16644606

Skiba, R. J., Simmons, A. B., Ritter, S., Gibb, A. C., Rausch, M. K., Cuadrado, J. (2008). Achieving equity in special education: History, status, and current challenges. Exceptional Children, 74(3), 264-288. 
Skiba, R. J., Poloni-Staudinger, L., Gallini, S., Simmons, A. B., \& Feggins-Azziz, R. (2006). Disparate access: The disproportionality of African American students with disabilities across educational environments. Exceptional Children, 72(4), 411-424.

*Skiba, R., Simmons, A., Ritter, S., Kohler, K., Henderson, M., \& Wu, T. (2006). The context of minority disproportionality: Practitioner perspectives on special education referral. Teachers College Record, (108)7, 1424-1459.

Stockman, I. J., Guillory, B., Seibert, M., \& Boult, J. (2013). Toward validation of a minimal competence core of morphosyntax for African American children. American Journal of Speech-Language Pathology, 22, 40- 56.

Sullivan, A. L., Artiles, A. J., \& Hernandez-Saca, D. I. (2015). Addressing SED inequity through systemic change: Contributions of ecologically based organizational consultation. Journal of Educational and Psychological Consultation, 25, 129-7. doi:10.1080/10474412.2014.929969

Terry, N. P. (2014). Dialect variation and phonological knowledge: Phonological representations and metalinguistic awareness among beginning readers who speak nonmainstream American English. Applied Psycholinguistics, 35, 155-176.

Terry, N. P., Connor, C. M., Johnson, L. S., Stuckey, A., \& Tani, N. (2016). Dialect variation, dialectshifting, and reading comprehension in second grade. Reading and Writing: An Interdisciplinary Journal, 29, 267-295.

Terry, N. P., Connor, C. M., Petscher, Y., \& Conlin, C. (2012). Dialect variation and reading: Is change in nonmainstream American English use related to reading achievement in first and second grade? Journal of Speech, Language, and Hearing Research, 55(1), 55-69. 
United States Department of Education, Office of Planning, Evaluation and Policy Development, Policy and Program Studies Service (2016). The State of Racial Diversity in the Educator Workforce. Washington, DC: Authors. Retrieved from:

https://www2.ed.gov/rschstat/eval/highered/racial-diversity/state-racialdiversity-workforce.pdf

Van Hofwegen, J., \& Wolfram, W. (2010). Coming of age in African American English: A longitudinal study. Journal of Sociolinguistics, 14(4), 427-455.

VERBI Software. (2019). MAXQDA 2020 [computer software]. Berlin, Germany: VERBI Software. Retrieved from: maxqda.com.

Wolfram, W. (2004). The grammar of urban African American Vernacular English, in Handbook of Varieties of English, (Eds). B. Kortmann and E. Schneider (Berlin: Moutonde Gruyter),111-132.

Young, V. A., Barrett, R., Young-Rivera, Y. \& Lovejoy, K. B. (2014). Other people's English: Code-meshing, code-switching, and African American literacy. New York, NY: Teachers College Press. 


\section{APPENDIX A: RECRUITMENT LETTER}

Principal Investigators: Camille Byrd O'Quin, M.A., CCC-SLP, Mark Zablocki, Ph.D., April Mustian, Ph.D., Debbie Shelden, Ph.D., Carrie Anna Courtad Ph.D., Lydia Kyei-Blankson. Ph.D.

To whom it may concern:

You are being asked to participate in a research study conducted by Mrs. Camille Byrd O’Quin, Doctoral Candidate in Special Education at Illinois State University; Dr. Mark Zablocki, Dr. April Mustian, Dr. Debbie Shelden, and Dr. Carrie Anna Courtad, Associate Professors in the Department of Special Education at Illinois State University; and Dr. Lydia Kyei-Blankson, Associate Professor in the Department of Educational Administration and Foundations at Illinois State University.

The purpose of this study is to ascertain in-service educators' perceptions of written work samples as they relate to special education referral.

\section{Why are you being asked?}

You have been asked to participate because you are an in-service educator, related personnel, or school administrator. Your participation in this study is voluntary. You will not be penalized if you choose to skip parts of the study, not participate, or withdraw from the study at any time.

\section{What would you do?}

If you choose to participate in this study, you will be expected to complete an online survey facilitated via Qualtrics. You will be asked 13 demographic questions (e.g., race/ethnicity, grade level working with, etc.); read four vignettes written by third graders and answer questions about referral for special education evaluation; and complete a 25-question survey using a 6-point Likert scale. You will then be asked if you would allow us to use your data in our research. In total, your involvement in this study will last approximately 15-20 minutes.

\section{Are any risks expected?}

We do not anticipate any risks beyond those that would occur in everyday life. To reduce these risks, you may skip any questions that make you uncomfortable or stop the survey at any time.

\section{Will your information be protected?}

Your responses will be anonymous; nothing that will identify you will be linked to your responses. The findings from this study may be presented in the form of presentations at state, national, or international conferences as well as research manuscripts for potential publication in scholarly journals.

\section{Who will benefit from this study?}

Benefits cannot be promised to participants from taking part in this research and choosing to have data included. There may be the added benefit of increased satisfaction in helping researchers understand more effective ways to teach in services educators about AAVE. 


\section{Whom do you contact if you have any questions?}

\section{Appendix A}

Recruitment Letter (continued)

If you have any questions about the research or wish to withdraw from the study, contact Mrs. Camille Byrd O'Quin by email at caoquin@ilstu.edu. You may also contact or Dr. Mark Zablocki, Associate Professor in the Department of Special Education at Illinois State University by email at mszablo@ilstu.edu.

If you have any questions about your rights as a participant, or if you feel you have been placed at risk, contact the Illinois State University Research Ethics \& Compliance Office at (309) 4385527 orIRB@ilistu.edu.

\section{Documentation of Consent}

If you are 18 or older and willing to participate in this study, provide electronic consent below by checking on the "Agree" button.

Agree

Disagree

You can print this form for your records.

Sincerely,

Mrs. Camille Byrd O’Quin, Doctoral Candidate Special Education Department Illinois State University 


\section{APPENDIX B: INFORMED CONSENT DEBRIEFING}

\section{Overrepresentation of African Americans in Special Education}

To whom it may concern:

\section{African American Vernacular English: Connecting the Dots to Disproportionality in Special Education}

Thank you for participating in this survey. This online survey was conducted to research educators' perceptions of work samples of African American Vernacular English (AAVE) versus Mainstream American English (MAE) and to analyze educators' perceptions of the AAVE dialect. This research study seeks to analyze the special education referral process and the possible connection to the overrepresentation of African Americans in special education. This was not revealed to you at the beginning of the survey as it may have influenced your responses.

\section{Data:}

The inclusion of your data as reported on this survey is voluntary. You may agree or disagree to allow us to use the data that you provided. Allowing your data to be used for this study is voluntary, and at any time you may opt to discontinue participation in this study.

All information is anonymous and reported results will be anonymized. Data will be stored on a password-protected device and/or locked filing cabinet. Anonymized demographic descriptors related to job description will be used in dissemination (e.g., special education teacher, speechlanguage pathologist, etc.). Your survey answers will be sent to a link at qualtrics.com where data will be stored in a password protected electronic format. Qualtrics will not collect identifying information such as your name, credentials, email address, or IP address.

\section{Research team contact information:}

For questions about this research, please contact: Mrs. Camille Byrd O'Quin by email at caoquin@ilstu.edu. You may also contact or Dr. Mark Zablocki, Associate Professor in the Department of Special Education at Illinois State University by email at mszablo@ilstu.edu.

Principal Investigators: Mrs. Camille Byrd O'Quin, Doctoral Candidate at Illinois State University; along with Dr. Mark Zablocki, Dr. April Mustian, Dr. Debbie Shelden, and Dr. Carrie Anna Courtad, Associate Professors in the Department of Special Education at Illinois State University; and Dr. Lydia Kyei-Blankson, Associate Professor in the Department of Educational Administration and Foundations at Illinois State University

\section{IRB contact about your rights in the study or to report a complaint:}

Research at Illinois State University involving human participants is carried out under the oversight of the Institutional Review Board (IRB). This research has been reviewed and approved by the IRB. If you have any questions about your rights as a participant, or if you feel 


\section{Appendix B}

Informed Consent Debriefing (continued)

you have been placed at risk, contact the Illinois State University Research Ethics \& Compliance Office at (309) 438-5527 or IRB@ilstu.edu.

\section{Withdrawing from the study:}

Participants may decide not to continue the research study at this time.

\section{Electronic Consent:}

Please select your choice below. You may print a copy of this consent form for your records. Clicking on the "Agree" button indicates that

- You voluntarily agree to have your data included in this research study

\section{Agree}

\section{Disagree}

Sincerely,

Mrs. Camille Byrd O'Quin,

Doctoral Candidate

Special Education Department

Illinois State University 


\section{APPENDIX C: PERCEPTION SURVEY}

Instructions: Please complete the following 25-question survey. Check one box for each statement that best represents your response regarding your perceptions of the dialects African American Vernacular English (AAVE) and Mainstream American English (MAE), two dialects spoken in the U.S. A 6-point Likert rating scale is used for this survey to include strongly disagree, disagree, slightly disagree, slightly agree, agree, and strongly agree.

\begin{tabular}{|c|c|c|c|c|c|c|}
\hline & $\begin{array}{l}\text { Strongly } \\
\text { Disagree }\end{array}$ & Disagree & $\begin{array}{l}\text { Slightly } \\
\text { Disagree }\end{array}$ & $\begin{array}{l}\text { Slightly } \\
\text { Agree }\end{array}$ & Agree & $\begin{array}{l}\text { Strongly } \\
\text { Agree }\end{array}$ \\
\hline $\begin{array}{l}\text { 1. AAVE is a misuse of } \\
\text { MAE. }\end{array}$ & & & & & & \\
\hline $\begin{array}{l}\text { 2. AAVE is a clear, } \\
\text { thoughtful, and } \\
\text { expressive language. }\end{array}$ & & & & & & \\
\hline $\begin{array}{l}\text { 3. AAVE has a faulty } \\
\text { grammar system. }\end{array}$ & & & & & & \\
\hline $\begin{array}{l}\text { 4. Continued usage of } \\
\text { AAVE would } \\
\text { accomplish nothing } \\
\text { worthwhile for society. }\end{array}$ & & & & & & \\
\hline $\begin{array}{l}\text { 5. Teachers should allow } \\
\text { African American } \\
\text { students to use AAVE in } \\
\text { the classroom. }\end{array}$ & & & & & & \\
\hline $\begin{array}{l}\text { 6. AAVE sounds as good as } \\
\text { MAE. }\end{array}$ & & & & & & \\
\hline 7. AAVE is cool. & & & & & & \\
\hline $\begin{array}{l}\text { 8. AAVE is as effective for } \\
\text { communication as is } \\
\text { MAE. }\end{array}$ & & & & & & \\
\hline $\begin{array}{l}\text { 9. If use of AAVE were } \\
\text { encouraged, speakers of } \\
\text { AAVE would be more } \\
\text { motivated to achieve } \\
\text { academically. }\end{array}$ & & & & & & \\
\hline $\begin{array}{l}\text { 10. In a predominantly } \\
\text { African American } \\
\text { school, AAVE as well as } \\
\text { MAE should be taught. }\end{array}$ & & & & & & \\
\hline $\begin{array}{l}\text { 11. Widespread acceptance } \\
\text { of AAVE is imperative. }\end{array}$ & & & & & & \\
\hline $\begin{array}{l}\text { 12. AAVE should be } \\
\text { considered a bad }\end{array}$ & & & & & & \\
\hline
\end{tabular}




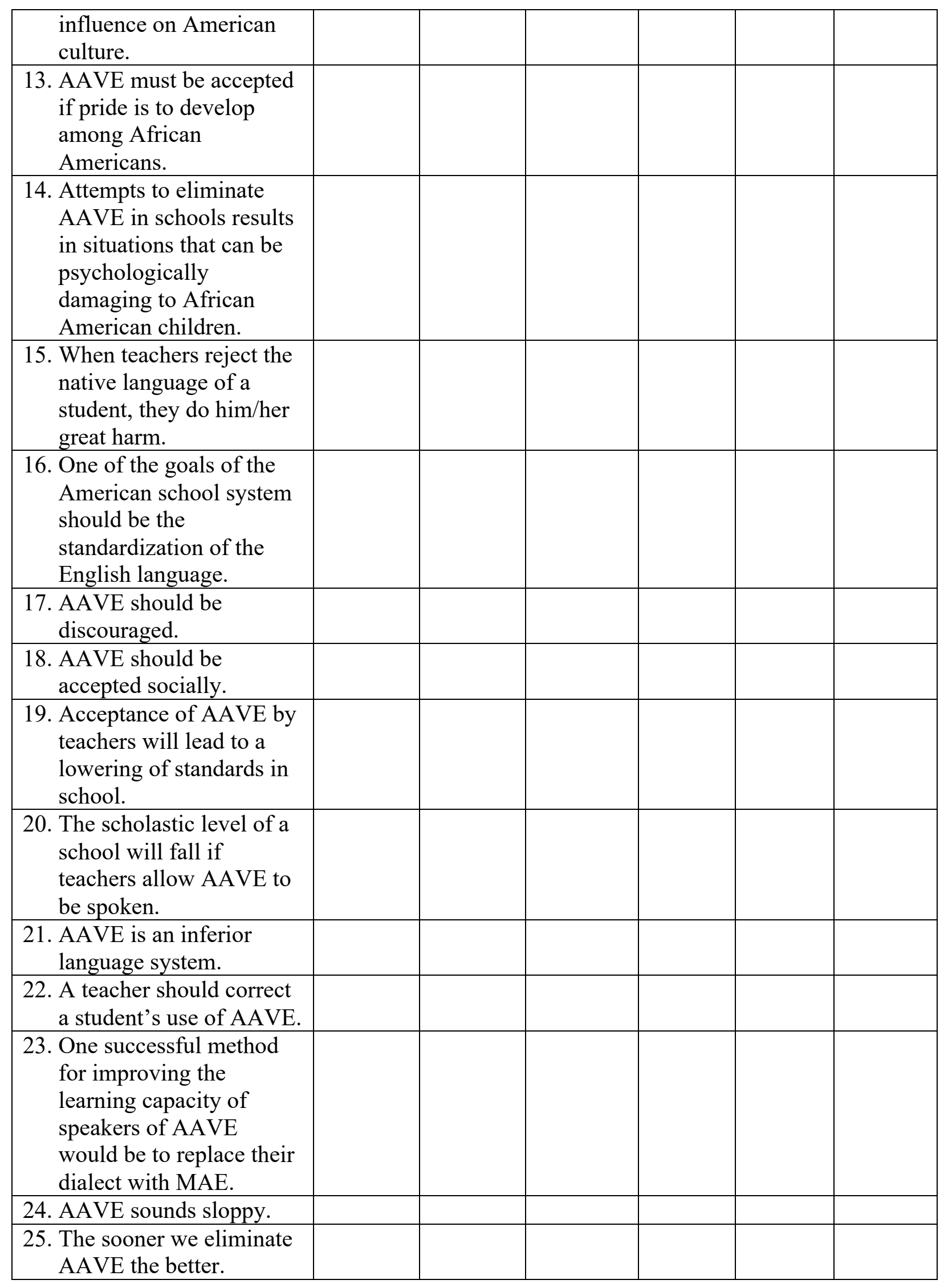




\section{APPENDIX D: DEMOGRAPHICS QUESTIONNAIRE}

Instructions: Please answer the following demographic question. Provide the response that best represents you. Check one or more boxes whenever applicable.

Current Role in Education (choose all that apply):

Educator

General education classroom teacher

Special education classroom teacher

Reading specialist/literacy coach

Special education resource teacher

Other

Related Personnel

School speech-language pathologist

School psychologist

School occupational therapist

School physical therapist

Case manager

School social worker

Other

School Administrator

Director of Special Education

Superintendent

Director of MTSS/RtI/PBIS

Director of Curriculum

Principal

Assistant Principal

Other

Grade Level currently working with (choose all that apply):

Pre-Kindergarten

Kindergarten - $2^{\text {nd }}$ grade

$3^{\text {rd }}$ grade $-5^{\text {th }}$ grade

$6^{\text {th }}$ grade $-8^{\text {th }}$ grade

$9^{\text {th }}-12^{\text {th }}$ grade

Prefer not to respond

Race/Ethnicity (choose all that apply):

Native American Indian or Alaskan

Asian

Black or African American

White or Caucasian

Native Hawaiian or other Pacific Islander

Hispanic or Latino 
Prefer not to respond

Other

Gender

Male

Female

Non-Binary

Gender-Nonconforming

Prefer not to respond

Other

Age Range

18-24 years

25-34 years

$35-44$ years

45-54 years

55-64 years

65+years

Prefer not to respond

Highest level of education completed:

High School Diploma

Associate's Degree

Bachelor's degree

Master's Degree

Doctorate

Prefer not to respond

Total years of experience teaching PreK-12:

$0-5$

6-10

11-15

$16-20$

$21+$

Prefer not to respond

Current PK-12 Educator Certification (choose all that apply):

Teacher certification/license

Provisional certification/license

Administration certification/license

Support personnel professional certification/license

Other

Not applicable

Prefer not to respond 
Public School where currently teaching Pre-K-12:

Teaching at Title I school

Teaching at non-Title I school

Not applicable

Prefer not to respond

Approximate \% of Black or African American students in attendance where you currently teach in a Prek-12 School:

$0-20$

$21-40$

$41-60$

$61-80$

$81-100$

Prefer not to respond

Number of Professional Development Courses you have completed that included objective(s) to address Reading-Language Arts:

$0-1$

$2-4$

$5-7$

$7+$

Prefer not to respond

Number of Professional Development Courses you have completed that included objective(s) to address Culturally Responsive Practices:

$0-1$

$2-4$

$5-7$

$7+$

Prefer not to respond

Number of Professional Development Courses you have completed that included objective(s) to address African American Vernacular English:

$0-1$

$2-4$

$5-7$

$7+$

Prefer not to respond 


\section{APPENDIX E: WRITTEN VIGNETTES}

Instructions: Please read the following four vignettes written by $3^{\text {rd }}$ grade students. Based on no other factors than these written vignettes and based solely on these superficial influences alone, indicate whether or not you would refer this student for a special education evaluation. After your response, you will be asked to provide a reason for why you did or why you did not refer this student for a special education evaluation.

\section{When My Puppies Ran Away}

One morning when I opened the back door, I expected my puppies, Maggie and Tucker, to jump up on me. But they didn't come at all. I called them, and they still didn't come. Now I knew something was wrong. I went and woke up my dad. He said, "Mom has it all under control." That's all I remembered about that night before I fell asleep.

The next day, I was still worried. I worried all day at school. When I got home, I asked my mom, "So, where are my puppies?" Her eyes started to fill with tears as she answered my question with 3 words, "I don't know." She burst into tears. I did, too. She hugged me and said, "If we never find them, I am sure they will have a good home." I sat on her lap and cried on her shoulder.

Yes, I would refer this student for a special education evaluation.

No, I would not refer this student for a special education evaluation.

Please briefly describe why you made the above choice.

\section{Amy and Oliva's Adventure}

Once there were two turtles. One was named Amy and one was Oliva. They were best friends. One day Amy decided they should go on an adventure. They tied a rope to each other so they couldn't separate. Then they decided to swim to the top of the ocean. When they stuck their heads out of the water, they saw a little island up ahead. "Let's go to it," said Oliva. "No! It could be dangerous!" said Amy. "I'll let you sunbathe!” said Oliva. "Let's go!” Amy said, rushing.

So, they swam to the island. When they got there, Amy laid down and started to sunbathe right away. But Oliva decided to collect some coconuts for lunch instead of sunbathing. "I'm hungry," said Amy. "Do we have any food?" "Yes, we do! I collected coconuts while you were sunbathing". "Let's start eating then!" said Amy.

Yes, I would refer this student for a special education evaluation.

No, I would not refer this student for a special education evaluation.

Please briefly describe why you made the above choice. 


\section{My Aunt}

My aunt use to live in Baltimore with my three cousin but last year she move to New York with my uncle. One night my aunt friend came over. My two cousin be sitting in the front room so that they can hear everything they say when they in the kitchen. My aunt and my aunt friend say they was going to spend the money she got for her birthday on some new shoes. But my uncle come in and say they paying bills.

I like to go to my aunt house because I like to play with the girl that live next door to they house. She been living there since she was 3. Last week I ask my friend that live next door to they house if she want to go see a basketball game with me and my two cousin. She said yes. So we all went up to the basketball game. That night my friend and my cousin ate until they was so full I thought they was going to bust.

Yes, I would refer this student for a special education evaluation.

No, I would not refer this student for a special education evaluation.

Please briefly describe why you made the above choice.

\section{A Boy Name Lester}

A boy name Lester live down the street from me. Lester is eight year old. Lester live next to his other friend Ollie. He like to play with Ollie and his two brother. Lester friend Ollie got into a fight with a older boy at school one day. Ollie don't like getting into fights. He promise the teacher that it wouldn't happen again so she wouldn't tell the principal. But his momma found out anyways and he got in big trouble.

But he was sick of being on punishment so he walk out of the house one day when his momma wasn't looking. He walk to my house to play ball. When his momma found out she came and got him and took him to his daddy house where it be so boring because he don't got no Xbox or nothing. He was there all weekend. When he got back to school he had a apple for the teacher and he look at me and smile.

Yes, I would refer this student for a special education evaluation.

No, I would not refer this student for a special education evaluation.

Please briefly describe why you made the above choice. 